\title{
A KdV-Fredholm Equation and Resonant Sloshing
}

\author{
by \\ John Michael Larratt MacNeil \\ Thesis submitted to the \\ Faculty of Graduate and Postdoctoral Studies \\ In partial fulfillment of the requirements \\ For the Master of Science degree in \\ Applied Mathematics
}

School of Mathematics and Statistics

Faculty of Science

Carleton University

(C) John Michael Larratt MacNeil, Ottawa, Canada, 2012 
Library and Archives

Canada

Published Heritage

Branch

395 Wellington Street

Ottawa ON K1A ON4

Canada
Bibliothèque et

Archives Canada

Direction du

Patrimoine de l'édition

395 , rue Wellington

Ottawa ON K1A ON4

Canada
Your file Votre référence

ISBN: 978-0-494-94319-9

Our file Notre référence

ISBN: 978-0-494-94319-9
NOTICE:

The author has granted a nonexclusive license allowing Library and Archives Canada to reproduce, publish, archive, preserve, conserve, communicate to the public by telecommunication or on the Internet, loan, distrbute and sell theses worldwide, for commercial or noncommercial purposes, in microform, paper, electronic and/or any other formats.

The author retains copyright ownership and moral rights in this thesis. Neither the thesis nor substantial extracts from it may be printed or otherwise reproduced without the author's permission.
AVIS:

L'auteur a accordé une licence non exclusive permettant à la Bibliothèque et Archives Canada de reproduire, publier, archiver, sauvegarder, conserver, transmettre au public par télécommunication ou par l'Internet, prêter, distribuer et vendre des thèses partout dans le monde, à des fins commerciales ou autres, sur support microforme, papier, électronique et/ou autres formats.

L'auteur conserve la propriété du droit d'auteur et des droits moraux qui protege cette thèse. $\mathrm{Ni}$ la thèse ni des extraits substantiels de celle-ci ne doivent être imprimés ou autrement reproduits sans son autorisation.
In compliance with the Canadian Privacy Act some supporting forms may have been removed from this thesis.

While these forms may be included in the document page count, their removal does not represent any loss of content from the thesis.
Conformément à la loi canadienne sur la protection de la vie privée, quelques formulaires secondaires ont été enlevés de cette thèse.

Bien que ces formulaires aient inclus dans la pagination, il n'y aura aucun contenu manquant. 


\begin{abstract}
The motion of a fluid with a free surface in a container subject to resonant forcing from the bottom and side is considered. The evolution of the free surface is shown to be governed by a forced $\mathrm{KdV}$ equation with an additional Fredholm integral term and is named the KdV-Fredholm equation. The steady-state of the forced KdV-Fredholm is then studied asymptotically in the limit of small dispersion. Three cases are considered, the forced $\mathrm{KdV}$, corresponding to the KdV-Fredholm without the Fredholm term; the forced $\mathrm{KdV}$ with a weak Fredholm term; and the steady-state forced KdV-Fredholm equation. A generalized multiple scales approach is reviewed to study the forced $\mathrm{KdV}$ and the weak KdV-Fredholm equations. This approach is then combined with boundary layer analysis for integral equations to study the forced KdV-Fredholm. The analytic work is compared with numerical results.
\end{abstract}




\section{Acknowledgements}

The author is first and foremost immeasurably grateful to his supervisor Professor Dave Amundsen. Few words can describe his bottomless patience, deep empathy and distinct humanity. I cannot imagine a better mentor.

A particular thank you is given to Professor Emmanuel Lorin for his help in nearly all my courses. Thank you for patiently introducing me to the world of abstractness, ingraining me with the concept of rigour and always being available.

These acknowledgements would be sorely incomplete without mentioning my friends who have encouraged me, laughed with me, and grounded me. A big thank you to the boys Max George-Lane, Jordan Bell and Tim Drager.

Finally, this thesis is dedicated to my entire family: James, Laura, John and Diana MacNeil. For everything. 


\section{Contents}

1 Introduction $\quad 1$

2 Governing Partial Differential-Integral Equation $\quad 4$

2.1 Introduction . . . . . . . . . . . . . . . . . . . . . 4

2.2 Mathematical Formulation . . . . . . . . . . . . . . 5

2.3 Steady-State Derivation . . . . . . . . . . . . . . . 9

2.3.1 Laplace's Equation . . . . . . . . . . . . . . . . . 9

2.3 .2 Surface Conditions . . . . . . . . . . . . . . . 10

2.4 The KdV-Fredholm Equation . . . . . . . . . . . . . . . . . . . 14

2.4.1 Influence of Vertical Forcing and The Mean Condition . . . . . 16

2.5 Dissipative Theory . . . . . . . . . . . . . . . 18

2.6 Summary ............................... 19

3 The Steady-State Forced KdV 20

3.1 Introduction . . . . . . . . . . . . . . . . . . 20

3.2 The Phase Plane . . . . . . . . . . . . . . . . 23

3.3 Asymptotic Assumptions and Modulation Theory . . . . . . . . . . 23

3.3.1 Asymptotic Assumptions . . . . . . . . . . . . . . . . . 23

3.3 .2 Modulation Theory . . . . . . . . . . . . . . 26

3.4 Non-dispersive solutions . . . . . . . . . . . . . . . . . . . . . . . . . . . . .

3.5 Dispersive Solutions . . . . . . . . . . . . . . . . . 28

3.5.1 Removing Secularity . . . . . . . . . . . . . . . . 29

3.5.2 The Resonant Band and Plotting . . . . . . . . . . . . . 32

3.5 .3 Nonlinear WKB Comment . . . . . . . . . . . . . . . 35

3.6 Effects of Dissipation . . . . . . . . . . . . . . . . . . . . . . . . . . . . . . . . . . .

3.7 Summary . . . . . . . . . . . . . . . . . 37 
4 The Weak KdV Fredholm Equation $\quad 38$

4.1 Introduction . . . . . . . . . . . . . . . . . 38

4.2 Non-dispersive and Dispersive Solutions . . . . . . . . . . . . . . . . 39

4.3 Solvability . . . . . . . . . . . . . . . . . . . 42

4.4 Comparison with Numerical Solutions . . . . . . . . . . . . . . . 46

4.5 Summary . . . . . . . . . . . . . . . . . . . 48

5 The Strong KdV-Fredholm $\quad 49$

5.1 Introduction . . . . . . . . . . . . . . . . . . . 49

5.2 A Nonlinear Fredholm Equation . . . . . . . . . . . . . . 50

5.2 .1 Non-dispersive Solutions . . . . . . . . . . . . . . 53

5.3 Boundary Layer Analysis . . . . . . . . . . . . . . . . . . 57

5.3 .1 Premlinaries ...................... 57

5.3 .2 Singularly Perturbed Volterra Equations . . . . . . . . . . 59

5.3 .3 Single Orbit Solutions . . . . . . . . . . . . . . 61

5.4 Multiple orbit Solutions . . . . . . . . . . . . . . 63

5.4.1 Removing Secularity and Physical Discussion ......... 64

5.5 Comparison with Numerical Results . . . . . . . . . . . . 66

6 Conclusion $\quad 69$

6.1 Summary of Results . . . . . . . . . . . . . . . . 69

6.2 Summary of Contributions . . . . . . . . . . . . . . . . . 69

6.3 Open Questions and Future Work . . . . . . . . . . . . . . . . 70

$\begin{array}{ll}\text { A Numerical Method } & 71\end{array}$ 


\section{List of Figures}

2.1 Illustration of the theoretical tank set up $\ldots \ldots \ldots \ldots \ldots$

3.1 Figure 1a) in Amundsen et al. illustrating the phase plane to the autonomous, constant F, system (3.8) [3]. . . . . . . . . . . . 24

3.2 Figure $2 \mathrm{a}$ ) and b) in Amundsen et al.[3] illustrating the various qualitative possibilities of the non-autonomous fKdV system within the boundary layer, ie. (3.8) where F now varies slowly. The fixed points are now the outer perturbative solutions to (3.7) allowing the trajectories (the rapidly varying inner solutions) to cross one another. . . . . . . . . . .

3.3 Figure 4 from Amundsen et al. [3] illustrating the norm of the inner solutions (in the diagram $u(x)$ in our work $z_{0}(x, X)$ for differing values of $\Delta$. Note the non-uniqueness of solutions for a given value of $\Delta . \ldots$.

3.4 Figure 3 from Amundsen et al. [3] illustrating the matching layer between the dispersive cnoidal solutions and non-dispersive solutions. To compare, we write the $u(x)$ in this figure as $z_{0}$ and $u_{ \pm}$as $y_{ \pm}$. A soliton solution, centred at $x^{*}$ is needed to match the cnoidal solutions to the non-dispersive solutions smoothly at the matching point $\tilde{x} . \ldots \ldots \ldots . \ldots 34$

3.5 Figure 6 from Amundsen et al. [3]. Notice that closed orbits no longer exist 36

4.1 Comparison of a double internal orbit asymptotic solution with numerical results for $c=2$. The steep peaks are given by the inner solutions $z_{0}(x, X)$ which are matched to the slowly varying middle is $y_{+}$. A full description of how the asymptotic solution was plotted is contained in Chapter Three. The dependent variable $x$ corresponds to real time at the steady-state and solution is plotted over one period. The parameter values are $\lambda=0.005$, $\nu=0.0236$. The value of $\Delta$ is 0.8786 , which falls within the resonant band. 47

5.1 Plot of $F(m)$ dictating $B_{+} \ldots \ldots \ldots \ldots \ldots \ldots \ldots$ 
5.2 Plot of $F_{2}(m)$ dictating $B_{-} \ldots \ldots \ldots \ldots \ldots \ldots$

5.3 Comparison of an undamped, single orbit soliton approximation $u=y_{+}+$ $z_{0}(x, X)$ with numerical solution for $c=1.5$. Parameter values are $\lambda=$ $0.005, \nu=1$ and $\Delta=1.8972$. A full description on how to plot $u(x)$ is given at the end of Chapter Three. . . . . . . . . . .

5.4 Comparison of an undamped, two orbit approximation $u(x)=y_{+}+$ $z_{0}(x, X)$ with numerical solution for $c=2$. Parameter values are $\lambda=$ $0.005, \nu=1$ and $\Delta=0.8370$. Note that the value of $\Delta$ for the double peak is smaller than the for the single peak plotted in Figure 5.3 and thus deeper within the resonant band. . . . . . . . . . . 68 


\section{Chapter 1}

\section{Introduction}

The notion of a liquid sloshing in a container is well known. It enters safety considerations for the design of almost any moving vehicle or structure containing liquid with a free surface including, but not limited too, tank-trucks, railway tank-cars, aircraft and transport ships [14]. In particular, sloshing in its most chaotic form, observed at resonance, must be accounted for. Resonant sloshing is most simply illustrated by imagining a tank with a piston acting at one end. The piston generates a wave and oscillates in such a way that the wave receives the same push every time it returns from reflecting at the closed end, eventually creating resonant effects. Resonant sloshing in a container has been studied since the first theoretical investigation conducted by Moiseyev [28] but there are still many aspects which are not well understood.

To understand the importance of sloshing, consider for a moment that $6.8 \%$ or 86.4 billion dollars of the Canadian GDP is attributed to energy and 2.2 billion tons of oil were transported by sea-faring oil tankers in 2006 [8, 34]. Furthermore, in 2006 the total displacement of the world fleet of sea-faring merchandise transporters was 960 million deadweight tons [34]. While there is no quantitative figure on how significantly inefficient design for sloshing can affect the world merchant fleet or transport of energy, we can say with a measure of confidence that even the slightest improvement could save substantial amounts of capital. This thesis does not intend contribute to such design improvements, nor is it directed at saving capital. This work is a mathematical investigation of sloshing and the theoretical system under consideration is necessarily idealized. The figures serve to show that in an industry with such extensive economic influence, analytic mathematical development for even highly idealized theory in the analysis of sloshing is a potentially valuable contribution. Idealized theory, and the 
mathematical development necessary for its analysis, provides the foundation for further and more relevant generalizations. This theoretical and mathematical development is our motivation.

Broadly speaking, there are three types of sloshing in rectangular containers that have dominated research since Moiseyev [28]. The first, which we will call surging, is precisely the example we illustrated above to describe sloshing, where the side is forced resonantly. The second, pitching, is when the container tips back and forth at the resonant frequency of the wave and the final type is simply a combination of both. Moiseyev's study considered surging for a relatively deep volume of fluid. Chester [9] and Chester and Bones [10] then conducted an independent study developing a steadystate sloshing theory for surging tanks and corroborated this experimentally. Resonant surface waves in general were considered by Ockendon and Ockendon [30] in a number of different scenarios including resonant sloshing, the case of shallow water surging being treated more thoroughly in [31]. This again, however, was a derivation of the steadystate equation and the full evolution for the shape of shallow water surging waves was given by Cox and Mortell [12] in the form of a forced Korteweg-deVries (fKdV) equation. This equation was in turn studied analytically by Amundsen et al. [3]. More recently, experimental work on two-dimensional surging waves has been performed by Kobine [23] which exhibited characteristics analogous to those observed by Chester and Bones [10]. We note that in each theoretical surging study, a single closed form differential equation amenable to asymptotic analysis was found, i.e. the theory was analytically tractable. Pitching has been considered most notably by Faltinsen and Timokha [13] and their findings indicate that there are significant challenges associated with analyzing the phenomena. The challenge we are primarily concerned with is the analytic intractability of the asymptotic theory of pitching which is, at present, largely studied numerically [13].

Intuitively, there are forcing elements in pitching which can be attributed to surging forcing as well as varying the height or gravity of the container. To theoretically explore these forcing elements, this thesis considers the motion of a shallow fluid in a container undergoing horizontal forcing as well as vertical forcing from the bottom. It is by examining these effects exclusively, while ignoring rotational effects, that we take a natural first step in analytically bridging the theories of surging and pitching.

The case of vertically forced fluid surfaces has been studied in connection with air motion and water waves [30]. The scenario of combining forcing at the bottom and side of the tank has, to the author's knowledge, not been considered. To pursue an analytical 
bridge between surging and pitching, we look to determine an asymptotic, analytically tractable, model equation describing the system and develop the necessary mathematical tools for studying this equation.

We now outline the organization of this thesis. In Chapter Two, we use the derivation technique of Cox and Mortell [12] to show that a resonantly forced KdV-Fredholm equation governs the evolution of the location of the free surface of an inviscid fluid in a tank undergoing vertical and horizontal forcing. Dissipation is then incorporated in the form of a Burger's damping term and justification for this is provided. The equation, to the author's knowledge, is of a novel type and the logic behind its naming is also described. The steady-state of the forced KdV-Fredholm equation

$$
\lambda u^{\prime \prime}(x)=\frac{3}{4} u^{2}(x)+\nu \int_{-1}^{1} \cos \pi(x+v) u(v) d v+\mu u^{\prime}+\cos (\pi x)-c
$$

is then studied asymptotically in the limit $\lambda \ll 1$ in the subsequent chapters. Chapter Three studies (1.1) with $\nu=0$, recovering the steady-state fKdV and amounts to a review of the work of Amundsen et al. [3] with a comment on nonlinear WKB theory. In Chapter Four, the integral term is introduced weakly and studied in a manner similar to the fKdV. In Chapter Five, the equation is studied with the integral term at the same order as the horizontal forcing. To do so, a method for asymptotically studying integral equations attributed to Angell and Olmstead $[4,5]$ is briefly reviewed and subsequently combined with the analysis of Amundsen et al. [3]. The work is effectively self-contained, the relevant background consisting of standard perturbative techniques. 


\section{Chapter 2}

\section{Governing Partial Differential-Integral Equation}

\section{$2.1 \quad$ Introduction}

The complete water-wave problem given in the next section is, in general, very difficult to solve numerically let alone analytically. By and large, the field of wave modelling relies heavily on seeking approximate equations to this based on small parameters that dictate the particular regime of interest, e.g. shallow water, deep water or small amplitude. The predictions based upon these approximate equations or systems of equations tend to agree very well with the experimentally observed phenomena of interest making them an attractive analytic tool in studying the full wave problem.

As described in the introduction and motivation, Chester and Bones [10] as well as Ockendon and Ockendon [31] derived closed form ordinary differential equations describing horizontally resonantly forced shallow waves in a tank. This, however, only described the steady state of the wave and soon after, the question of how the wave evolved was answered by Cox and Mortell [12]. The equation modelling the evolution of the wave undergoing surging was a periodically forced KdV equation. Cox and Mortell [12] arrived at the equation by expanding the velocity potential and the location of the free surface in an asymptotic series with the small parameters being the amplitude of the forcing and the aspect ratio of the undisturbed height of the fluid to the length of the tank. There are of course, other avenues one can follow when deriving approximate equations. A recent technique, known as modal analysis, involves expanding the velocity potential and location of the surface into their Fourier modes and deriving a system of ODE's 
describing how these modes evolve $[32,13]$. These systems tend to be strongly nonlinear, coupled ODE's which resist analytic treatment and are largely solved numerically. It is our intention to put nonlinear sloshing with bottom forcing or resonant gravitational effects, into an analytically tractable setting. In this pursuit, we will be closely following the technique of Cox and Mortell [12] although slightly adjusted to find the governing evolution equation of a wave in a tank whose bottom and side are being resonantly forced.

In this chapter we show evolution of the free surface is governed by a periodically forced KdV equation with a Fredholm integral term. This equation, to the author's knowledge, is of a novel type and the nomenclature is justified within in chapter. In Section 2.2, the full mathematical formulation of the problem with the accompanied asymptotic assumptions is described. In Section 2.3 the steady-state integro-differential equation is derived. In Section 2.4 the full forced KdV-Fredholm equation is derived and finally in Section 2.5 the inclusion of dissipation is discussed.

\subsection{Mathematical Formulation}

The system under consideration is the irrotational motion of an invisicid, incompressible, homogeneous fluid of constant density $\rho_{0}$ subject to a constant gravitational force with acceleration $g$. The fluid is initially at rest with an undisturbed height $H$ contained in a rectangular tank of length $L$ which is closed and impermeable at one end, $x=0$, and has a pair of idealized oscillatory wave makers. One wave-maker has a maximum amplitude $l$ located at $x=L-l \cos (\omega t)$ and corresponds to a purely horizontal forcing. The other has an amplitude of $h$ located at $y=-H+h \cos \left(\omega_{2} t\right)$ and corresponds to a vertical forcing with a different frequency.

Within the fluid body domain $\Gamma(\eta)=\{(x, y): 0<x<L-l \cos (\omega t),-H+$ $\left.h \cos \left(\omega_{2} t\right)<y<\eta(x, t)\right\}$ where $\eta(x, t)$ is the location of the surface, the velocity potential of the fluid $\phi(x, y, t)$ satisfies Laplace's equation,

$$
\phi_{x x}+\phi_{y y}=0 \text {. }
$$

As the tank is closed and immobile at $x=0, \phi(x, y, t)$ is subject to the boundary condition

$$
\phi_{x}=0 \quad \text { at } x=0
$$

The wave makers dictate the fluid velocity normal to their respective portions of the boundary implying

$$
\phi_{x}=\omega l \sin (\omega t) \quad \text { at } x=L-l \cos (\omega t),
$$




$$
\phi_{y}=-\omega_{2} h \sin \left(\omega_{2} t\right) \quad \text { at } y=-H+h \cos \left(\omega_{2} t\right)
$$

Along the surface $y=\eta(x, t)$, the kinematic and dynamic conditions apply and read, respectively,

$$
\begin{gathered}
\eta_{t}+\phi_{x} \eta_{x}-\phi_{y}=0 \\
\phi_{t}+\frac{1}{2}\left(\phi_{x}^{2}+\phi_{y}^{2}\right)+g \eta=0
\end{gathered}
$$

where $g$ is the gravitational constant. Finally, the initial conditions correspond to that of a fluid at rest and a forcing beginning just after $t=0$, such that

$$
\left.\begin{array}{c}
\eta=\eta_{t}=0 \\
\phi_{x}=\phi_{y}=0
\end{array}\right\} \quad \text { for } t \leq 0,-H<y<0,0 \leq x \leq L .
$$

To derive the closed form equation governing the system of interest, we make the following assumptions,

- The undisturbed height of the fluid is small with respect to the length of the tank.

- The amplitude of the horizontal forcing is small with respect to the length of the tank.

- The amplitude of the vertical forcing is small with respect to the undisturbed height of the fluid.

These assumptions lead naturally to three small dimensionless parameters which characterize the motion of the fluid,

$$
\begin{gathered}
\delta=\frac{H}{L} \ll 1, \\
\varepsilon=\frac{l}{L} \ll 1, \\
\gamma=\frac{h}{H} \ll 1 .
\end{gathered}
$$

To introduce the appropriate asymptotics and illuminate the impact of these quantities, we non-dimensionalize the above system by introducing the variables,

$$
\phi^{\prime}=\frac{\phi}{c_{0} L}, \quad t^{\prime}=\frac{\omega t}{\pi}, \quad \eta^{\prime}=\frac{\eta}{H}, \quad x^{\prime}=\frac{x}{L}, \quad \omega^{\prime}=\frac{\omega L}{2 \pi c_{0}} .
$$

Here, $c_{0}=\sqrt{g H}$ is the long-wave speed and we take the bottom forcing to be some integer multiple of the side forcing or $\omega_{2}=\alpha \omega$ for $\alpha \in \mathbb{Z}$, to ensure periodicity of the forcing. It 


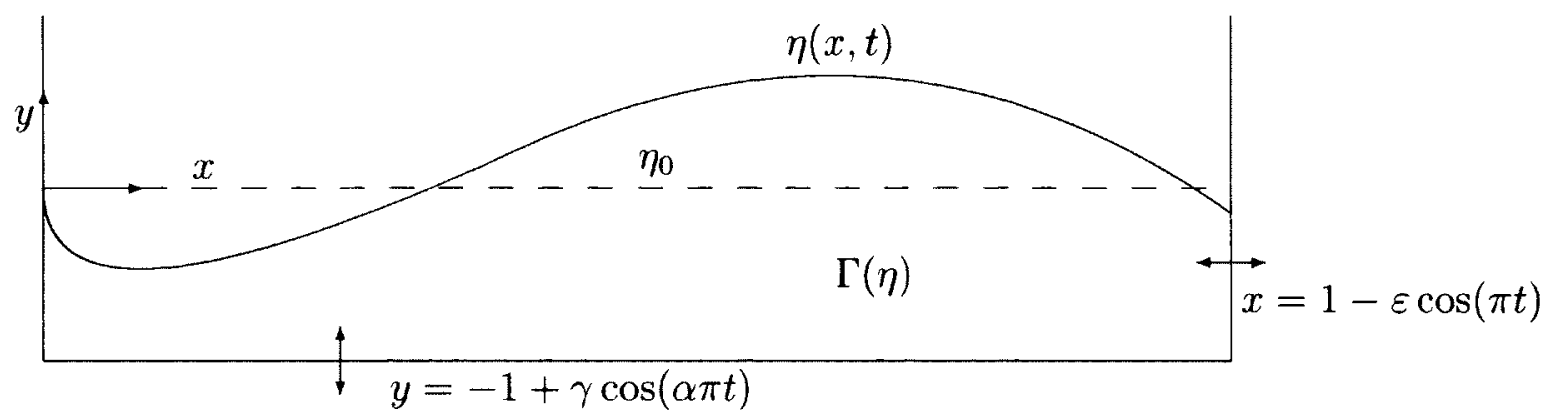

Figure 2.1: Illustration of the theoretical tank set up

should be noted here that the linear wave speed is taken as that of a wave in shallow water of constant depth rather than a variable one. This is justified by the eventual expansion of the bottom boundary condition around $y=-H$, mathematically fixing the bottom and therefore maintaining the leading order wave speed. After dropping the primes, the above change of variables transforms (2.1)-(2.6) to the following dimensionless system.

$$
\begin{gathered}
\delta^{2} \phi_{x x}+\phi_{y y}=0 \quad \text { for }(x, y) \in \Gamma(\eta), \\
\phi_{x}=0 \quad \text { at } x=0, \\
\phi_{x}=2 \pi \varepsilon \omega \sin (\pi t) \quad \text { at } x=1-\varepsilon \cos (\pi t), \\
\phi_{y}=-2 \pi \gamma \alpha \omega \sin (\alpha \pi t) \quad \text { at } y=-1+\gamma \cos (\alpha \pi t), \\
\phi_{y}-\delta^{2}\left(2 \omega \eta_{t}+\phi_{x} \eta_{x}\right)=0, \\
2 \omega \phi_{t}+\frac{1}{2}\left(\phi_{x}^{2}+\delta^{-2} \phi_{y}^{2}\right)+\eta=0,
\end{gathered}
$$

and the initial resting fluid conditions remain unchanged. Figure 2.1 illustrates the non-dimensionalized theoretical framework.

In order to seek an asymptotic approximation to (2.7)-(2.13), some relationship between $\delta, \varepsilon$ and $\gamma$ is needed. Ockendon and Ockendon [30] show that for $\mathcal{O}(\varepsilon)$ horizontal 
forcing, the amplitude response is $\mathcal{O}\left(\varepsilon^{\frac{1}{2}}\right)$ which can be captured by adopting the similarity relation

$$
\delta^{2}=\kappa \varepsilon^{\frac{1}{2}},
$$

where $\kappa$ is $\mathcal{O}(1)$ in $\varepsilon$. In the absence of vertical forcing, this eliminates the leading order dependence of $\phi(x, y, t)$ on $y$ and eventually strikes a balance between nonlinear and dispersive effects which become dominant over the linear acoustic growth as the amplitude of the wave transitions from $\mathcal{O}(\varepsilon)$ to $\mathcal{O}\left(\varepsilon^{\frac{1}{2}}\right)$. The justification for this scaling was founded in the experimental work of Chester and Bones [10] who recorded a balance between dispersion and nonlinearity which manifested in soliton like entities that arouse near and at resonance. The present study is a highly idealized, fictitious system and as a result direct experimental comparison is presently unavailable. Our goal of finding an analytically tractable model, however, remains unchanged. In particular, we wish to theoretically explore coupling effects between the vertical and the horizontal forcing. Intuitively, strong coupling would arise only if the magnitude of the bottom forcing is either larger or equivalent to the horizontal forcing, thus we can impose the similarity relation on $\gamma$ to be

$$
\gamma=\beta \varepsilon,
$$

where $\beta$ is $\mathcal{O}(1)$ in $\varepsilon$. To analytically develop the theory of sloshing forward in the most natural manner, we adopt the same similarity relation as Cox and Mortell and Ockendon and Ockendon $[12,30]$ for the aspect ratio given by $(2.14)$. This is a reasonable direction of study. We are still anticipating a resonant response from the bottom forcing of $\mathcal{O}\left(\varepsilon^{\frac{1}{2}}\right)$ but we are overlooking the possibility of violent behaviour characteristic of pitching normally found with similarity relations such as $\delta^{2}=\tilde{\kappa} \varepsilon^{\frac{1}{3}}$ or $\delta^{2}=\tilde{\kappa} \varepsilon^{\frac{1}{4}}$ [13]. Here we are forced to rely on intuition to justify this. The particularly violent behaviour discussed by Faltinsen and Timohka [13] is experimentally verified as a result of the angular motion of the tank. This angular motion would, in all likelihood, accelerate the wave speed as it approaches the walls causing run-up or breaking. In our formulation, the wave speed effectively remains constant as commented on in the next section, so in the absence of such acceleration we would assume the scaling adopted from Ockendon and Ockendon [30] to be reasonable. 


\subsection{Steady-State Derivation}

Using these scalings, $\phi(x, y, t)$ and $\eta(x, t)$ can be expanded in a power series of $\varepsilon$ while making a small amplitude assumption, as follows

$$
\begin{gathered}
\phi(x, y, t)=\varepsilon^{\frac{1}{2}} \phi_{0}(x, y, t)+\varepsilon \phi_{1}(x, y, t)+\varepsilon^{\frac{3}{2}} \phi_{2}(x, y, t)+\mathcal{O}\left(\varepsilon^{2}\right), \\
\eta(x, t)=\varepsilon^{\frac{1}{2}} \eta_{0}(x, t)+\varepsilon \eta_{1}(x, t)+\varepsilon^{\frac{3}{2}} \eta_{2}(x, t)+\mathcal{O}\left(\varepsilon^{2}\right) .
\end{gathered}
$$

The choice to begin the series at $\varepsilon^{\frac{1}{2}}$ rather than at $\varepsilon$ is discussed in detail at the end of the derivation. The technique of Cox and Mortell [12] we will be following can be summarized by:

1. Solving Laplace's equation with linear functions of $y$ and arbitrary functions $\theta_{i}(x, t)$.

2. Using these solutions to derive successive wave equations for the functions $\theta_{i}(x, t)$ on the surface.

3. Solving these wave equations with general solutions such that they satisfy the boundary condition at $x=0$

4. Impose the boundary condition to this general solution at $x=1$ to obtain a integrodifferential equation.

\subsubsection{Laplace's Equation}

Substituting the expansion for $\phi(x, y, t)$ into Laplace's equation gives for the first few orders,

$$
\begin{aligned}
& \mathcal{O}\left(\varepsilon^{\frac{1}{2}}\right) \rightarrow \phi_{0 y y}=0 \\
& \mathcal{O}(\varepsilon) \rightarrow \phi_{1 y y}=-\kappa \phi_{0 x x} \\
& \mathcal{O}\left(\varepsilon^{\frac{3}{2}}\right) \rightarrow \phi_{2 y y}=-\kappa \phi_{1 x x}
\end{aligned}
$$

As we will find in a moment, the leading order acoustic wave speed is just one, implying a resonant frequency $\omega=\frac{1}{2}$. With this in mind we introduce a detuning parameter $\Delta \in \mathbb{R}$ such that

$$
\varepsilon^{\frac{1}{2}} \Delta=2 \omega-1 .
$$

Note that by taking the forcing frequency to be the fundamental frequency $\omega=\frac{1}{2}$ the detuning parameter $\Delta$ simply becomes zero. As experimenters will generally resonantly force a tank at differing values $\omega$ to explore different behaviour (see [13]), $\Delta$ as we have 
introduced is a practical artifact to facilitate the connections of this work to experiment by allowing us to mathematically vary the frequency. The power of epsilon predicts the existence of a resonant band of width $\varepsilon^{\frac{1}{2}}$ in which nonlinear and dispersive effects are pertinent, and thus by "tuning" $\Delta$ all possible behaviour in the band can be explored. Substituting the relation between $\gamma$ and $\varepsilon$ as well as $2 \omega=\varepsilon^{\frac{1}{2}} \Delta+1$ into the bottom boundary condition (2.11) and expanding around around $y=-1$ gives,

$$
\begin{gathered}
\mathcal{O}\left(\varepsilon^{\frac{1}{2}}\right) \rightarrow \phi_{0 y}=0, \\
\mathcal{O}(\varepsilon) \rightarrow \phi_{1 y}=-\beta \alpha \pi \sin (\alpha \pi t), \\
\mathcal{O}\left(\varepsilon^{\frac{3}{2}}\right) \rightarrow \phi_{2 y}=-\Delta \beta \alpha \pi \sin (\alpha \pi t) .
\end{gathered}
$$

The consecutive Laplace's equations with their respective boundary conditions can be solved to get,

$$
\begin{aligned}
& \phi_{0}=\theta_{0}(x, t), \\
& \phi_{1}=\theta_{1}(x, t)-\kappa\left(y+\frac{1}{2} y^{2}\right) \theta_{0 x x}-\pi \beta \alpha y \sin (\alpha \pi t) . \\
& \phi_{2}=\theta_{2}(x, t)-\kappa\left(y+\frac{1}{2} y^{2}\right) \theta_{1 x x}+\kappa^{2}\left(-\frac{1}{3} y+\frac{1}{6} y^{3}+\frac{1}{24} y^{4}\right) \partial_{x}^{4} \theta_{0}-\Delta \pi \beta \alpha y \sin (\alpha \pi t),
\end{aligned}
$$

Note that these solutions hold for $t>0$, as for $t \leq 0$ the forced boundaries are immobile.

\subsubsection{Surface Conditions}

Substituting the expansion for $\phi(x, y, t)$ and $\eta(x, t)$ as well as the parameters $\gamma, \delta$ and $\omega$ in terms of $\varepsilon$ into the dynamic condition (2.13) yields at the first few orders,

$$
\begin{aligned}
\mathcal{O}\left(\varepsilon^{\frac{1}{2}}\right) & \rightarrow \phi_{0 t}+\eta_{0}=0, \\
\mathcal{O}(\varepsilon) & \rightarrow \phi_{1 t}+\Delta \phi_{0 t}+\eta_{1}+\phi_{0 x}^{2}=0
\end{aligned}
$$

Similarly the first few orders for the kinematic condition (2.12) are

$$
\begin{array}{ccc}
\mathcal{O}(\varepsilon) & \rightarrow & \phi_{1 y}-\kappa \eta_{0 t}=0, \\
\mathcal{O}\left(\varepsilon^{\frac{3}{2}}\right) & \rightarrow & \phi_{2 y}-\kappa\left(\eta_{1 t}+\Delta \eta_{0 t}+\eta_{0 x} \phi_{0 x}\right)=0 .
\end{array}
$$

The expressions found for the respective functions $\phi_{i}$ substituted into (2.21)-(2.24) will yield a wave equation for both $\theta_{0}$ and $\theta_{1}$. While the details of this are simple, they are not immediately obvious and deserve to be shown in full for the derivation of the $\theta_{0}(x, t)$ wave equation. Rearranging (2.21) for $\eta_{0}$, substituting the expression for $\phi_{0}$ and differentiating in $t$ gives,

$$
\eta_{0 t}=-\theta_{0 t t}(x, t) .
$$


Substituting this and the expression for $\phi_{1}$ into the kinematic condition (2.23) gives

$$
\begin{gathered}
\left.\partial_{y}\left\{\theta_{1}(x, t)-\kappa\left(y+\frac{1}{2} y^{2}\right) \theta_{0 x x}-\pi \beta \alpha y \sin (\alpha \pi t)\right\}\right|_{y=0+\varepsilon^{\frac{1}{2}}} \eta_{0}+\ldots+\kappa \theta_{0 t t}=0 \\
\Rightarrow-\kappa\left(\theta_{0 x x}-\theta_{0 t t}\right)-\varepsilon^{\frac{1}{2}} \kappa \eta_{0} \theta_{0 x x}=\alpha \beta \pi \sin (\alpha \pi t)
\end{gathered}
$$

Thus the leading order wave equation for $\theta_{0}$ is

$$
\theta_{0 x x}-\theta_{0 t t}=-\frac{\alpha \beta \pi}{\kappa} \sin (\alpha \pi t)
$$

The nonlinearity $\varepsilon^{\frac{1}{2}} \kappa \eta_{0} \theta_{0 x x}$ is carried into the kinematic condition at $\mathcal{O}(\varepsilon)$. The same substitutions and arrangements can be made to find the wave equation for $\theta_{1}(x, t)$,

$$
\theta_{1 x x}-\theta_{1 t t}=2 \Delta \theta_{0 t t}-\frac{1}{3} \kappa \partial_{x}^{4} \theta_{0}+2 \theta_{0 x} \theta_{0 x t}+\theta_{0 t} \theta_{0 x x}-\frac{\Delta \beta \alpha \pi}{\kappa} \sin (\pi t)
$$

We now turn to solving the wave equations in general and applying the boundary conditions. Solving the governing equation for $\theta_{0}$ in general yields

$$
\theta_{0}=f(x+t-1)+g(t-x)-\frac{\beta}{\kappa \alpha \pi} \sin (\alpha \pi t)
$$

and applying the boundary condition (2.9) at the immobile left wall gives

$$
f^{\prime}(t-1)=g^{\prime}(t)
$$

The boundary condition (2.10) implies that

$$
f^{\prime}(t)=f^{\prime}(t-2)
$$

providing us with the so-called "acoustic representation" [12] of the velocity potential given by

$$
\theta_{0}=f(x+t-1)+f(t-x-1)-\frac{\beta}{\kappa \alpha \pi} \sin (\alpha \pi t)
$$

where $f$ is an initially even, arbitrary, 2-periodic function. Note that the initial evenness satisfies the initial conditions. We can see that the sinusoidal term is the linear response of the system to the bottom forcing in the absence of an initial wave. To fully determine the general function $f$ we move to the second order approximation of $\phi(x, y, t)$ retaining the representation $(2.27)$ of $\theta_{0}(x, t)$ for simplicity. Solving for the particular solution of (2.26) yields

$$
\begin{array}{r}
\theta_{1}(x, t)=-\frac{1}{6} \kappa x\left\{f^{\prime \prime \prime}(x+t-1)-g^{\prime \prime \prime}(t-x)\right\}+\Delta x\left\{f^{\prime}(x+t-1)-g^{\prime}(t-x)\right\} \\
+\frac{1}{4}\left\{f(x+t-1) g^{\prime}(t-x)+g^{\prime}(t-x) f(x+t-1)\right\}+\frac{\beta}{\alpha \pi \kappa} \Theta\left(f^{\prime \prime}, g^{\prime \prime}\right) \\
\left.+\frac{3}{4} x\left\{f^{\prime}(x+t-1)^{2}-g^{\prime}(t-x)^{2}\right)\right\}+\frac{\Delta \beta}{\alpha \pi \kappa} \sin (\alpha \pi t)
\end{array}
$$


where

$\Theta\left(f^{\prime \prime}, g^{\prime \prime}\right)=\int_{0}^{x} \sin (\alpha \pi(s-x+t)) f^{\prime \prime}(2 s-x+t-1)-\sin (\alpha \pi(x-s+t)) g^{\prime \prime}(x-2 s+t) d s$.

Note that using the relation (2.28) this expression also satisfies the boundary condition at the left side of the tank (2.9). Applying the entire expansion of $\phi(x, y, t)$ to satisfy the right boundary condition, we adopt the representation

$$
\phi_{x}=\varepsilon^{\frac{1}{2}} \theta_{0 x}+\varepsilon \theta_{1 x}+\ldots
$$

as $\phi_{i x}=\theta_{i x}$ for $y=0+\varepsilon^{\frac{1}{2}} \eta_{0}+\ldots$ Letting $h(t)=f^{\prime}(t)$ we truncate at $\theta_{2}$ to get the full delayed-integro-differential equation,

$$
\begin{aligned}
\varepsilon \pi \sin (\pi t)= & \varepsilon^{\frac{1}{2}}\{h(t)-h(t-2)\} \\
& +\varepsilon\left\{-\frac{1}{6} \kappa\left\{h^{\prime \prime}(t)-h^{\prime \prime}(t-2)\right\}-\frac{1}{6} \kappa\left\{h^{\prime \prime \prime}(t)+h^{\prime \prime \prime}(t-2)\right\}\right. \\
& +\Delta\{h(t)-h(t-2)\}+\Delta\left\{h^{\prime}(t)+h^{\prime}(t-2)\right\} \\
& \frac{3}{4}\left\{h^{2}(t)-h^{2}(t-2)\right\}+\frac{3}{2}\left\{h(t) h^{\prime}(t)+h(t-2) h^{\prime}(t-2)\right\} \\
+ & \left.\frac{1}{4}\left\{h^{\prime}(t-2) \int^{t} h(s) d s-h^{\prime}(t) \int^{t-2} h(s) d s\right\}+\left.\tilde{\beta} \partial_{x} \Theta(h(t))\right|_{x=1}\right\},
\end{aligned}
$$

where $\tilde{\beta}=\frac{\beta}{\alpha \kappa \pi}$ and

$$
\begin{aligned}
\left.\partial_{x} \Theta\right|_{x=1} & =\sin (\alpha \pi(t-1)) h^{\prime}(t)-\sin (\alpha \pi(t-1)) h^{\prime}(t-2) \\
& +\int_{0}^{1} \sin \left(\alpha \pi(t+s-1) h^{\prime \prime}(t+2 s-2)+\sin (\alpha \pi(t-s+1)) h^{\prime \prime}(t-2 s) d s .\right.
\end{aligned}
$$

The relation, $h(t)=h(t-2)$ from (2.29) implies we are seeking solutions with the same period as the driver and leaves the integro-differential equation,

$$
\frac{3}{2} h(t) h^{\prime}(t)+\Delta h^{\prime}(t)-\frac{\kappa}{6} h^{\prime \prime \prime}(t)+\tilde{\beta} \tilde{\Theta}\left(h^{\prime \prime}(t)\right)=\frac{\pi}{2} \sin (\pi t),
$$

where

$$
\tilde{\Theta}\left(h^{\prime \prime}(t)\right)=\int_{0}^{1} \sin \left(\alpha \pi(t+s-1) h^{\prime \prime}(t+2 s)+\sin (\alpha \pi(t-s+1)) h^{\prime \prime}(t-2 s) d s .\right.
$$


Note this agrees with Cox and Mortell [12] for zero vertical forcing, ie. when $\tilde{\beta}=0$. There is a step in methodology of Cox and Mortell [12] we have skipped. At the end of their analysis, $h(t)$ is re-scaled by a power of $\varepsilon^{\frac{1}{2}}$. This serves to match the dispersive and nonlinear effects to the same order of $\varepsilon$ as the external forcing. This is something already achieved in our delayed-integro-differential equation in the absence of such a rescaling. One can readily verify that re-scaling $h(t)=\varepsilon^{\frac{1}{2}} \tilde{h}(t)$ and taking $\tilde{\beta}=0$ recovers the delayed-differential equation of Cox and Mortell [12]. The subtle difference in our technique is we anticipated this re-scaling by re-scaling the original ansatz, beginning the expansions at $\varepsilon^{\frac{1}{2}}$ rather that $\varepsilon$. This bypasses the acoustic response prediction that, before re-scaling, $h(t)=t \sin (\pi t)$. This acoustic response clearly becomes unbounded and thus the appropriate balance requires a re-scaling of $h(t)$. That we have recovered the same equation by re-scaling at the beginning shows there is a transitivity to this singular perturbation argument. In order words, in the absence of bottom forcing there is no difference between re-scaling the original series expansions and re-scaling $h(t)$ at the end of the derivation.

The necessity of using this transitivity in our work was to solve a troublesome illposedness problem. Supposing for a moment that we had taken the expansion for $\phi(x, y, t)$ to begin at $\mathcal{O}(\varepsilon)$ while leaving all other scalings and the system unaltered. The leading order system for $\phi_{0}$ would be the following

$$
\begin{gathered}
\phi_{0 y y}=0, \\
\phi_{0 y}=-\beta \alpha \pi \sin (\alpha \pi t), \quad y=-1, \\
\phi_{0 y}=0, \quad y=0+\varepsilon^{\frac{1}{2}} \eta_{0} .
\end{gathered}
$$

The first two obviously arise from Laplace's equation and the bottom boundary condition and the last from the kinematic condition (2.12). The leading order Laplace solution would still be a linear function of $y$ however, its "slope" function of $x$ and $t$ have to be equal to both the forcing on the bottom and zero on the surface. The implication here is if we do not incorporate the singular perturbation argument into the original expansions, we can not even begin a proper derivation. The discovery of this transitivity is of tremendous importance for the analysis of shallow water systems with bottom forcing. 


\subsection{The KdV-Fredholm Equation}

Returning to the derivation, we comment that the integro-differential equation in (2.34) represents the steady-state response of the system. Exercising a bit of physical intuition, one can imagine that waves gently forced from one side of the tank and the bottom do not immediately exhibit the nonlinear or dispersive behaviour but would ripple in a linear fashion. This notion has been corroborated by an abundance of experimental groups $[12,30,13]$ and implies that the acoustic response

$$
h(t)=\frac{\pi t}{2} \sin (\pi t)
$$

is valid while the wave height is $\mathcal{O}(\varepsilon)$. As long as the waves do not run-up or overturn, experiments show the evolution of the wave begins with with acoustic linear growth, tends towards a shock and eventually nonlinear and dispersive effects balance to avoid discontinuities [10]. The singular perturbation argument from the previous section dismissed the acoustic response on the grounds that it becomes unbounded. While reasonable, this neglects the validity of the acoustic response within a bounded time-frame. More specifically, the acoustic response is valid in the portion of the evolution when the amplitude is $\mathcal{O}(\varepsilon)$ until the amplitude reaches $\mathcal{O}\left(\varepsilon^{\frac{1}{2}}\right)$. From the full-delayed integro-differential equation (2.32) we can notice that the acoustic response arises from matching the $h(t)-h(t-2)$ term at $\mathcal{O}\left(\varepsilon^{\frac{1}{2}}\right)$ with the sinusoidal forcing, a balance that would require a re-scaling. To see this note that the solution to the difference equation

$$
h(t)-h(t-2)=\pi \sin \pi t
$$

is the acoustic response given by (2.36). The nonlinear and dispersive effects dominant at the steady-state arise from the balance already in place given in equation (2.34). It seems then, we have discretized the acoustic regime and the nonlinear/dispersive regime in our perturbative hierarchy. The final step in the derivation then is to incorporate the validity of the acoustic regime and track the transition when it interchanges dominance with the nonlinear/dispersive regime. To this end, we introduce a second time scale over which nonlinear effects become dominant. As $t$ given above reflects the amount of time for the wave to travel the length of the tank and back, this time-scale is retained and we introduce $\tau=\varepsilon^{\frac{1}{2}} t$ which reflects the time over which dispersive and nonlinear effects become substantial. Expanding $h(t)$ in terms of $\varepsilon$ such that, 


$$
h(t ; \varepsilon)=\sum_{j=0}^{N} \varepsilon^{j} h_{j}(t, \tau)
$$

The periodicity of $h(t)$ is maintained by setting $h_{i}(t, \tau)=h_{i}(t-2, \tau)$ for $i=0,1,2 \ldots$. Substituting this expansion for $h(t)$ into the delayed-integro-differential equation (2.32), dividing by $\varepsilon$ and noting the difference relation (see [12])

$$
h(t-2 ; \varepsilon)=h_{0}(t-2, \tau)-2 \varepsilon^{\frac{1}{2}} \frac{\partial h_{0}}{\partial \tau}(t-2, \tau)+\varepsilon h_{1}(t-2, \tau)+\ldots
$$

yields the governing forced KdV-Fredholm for $h_{0}$ after applying periodicity,

$$
\frac{\partial h_{0}}{\partial \tau}+\frac{3}{2} h_{0} \frac{\partial h_{0}}{\partial t}+\Delta \frac{\partial h_{o}}{\partial t}-\frac{\kappa}{6} \frac{\partial^{3} h_{0}}{\partial t^{3}}-\frac{\tilde{\beta} \alpha \pi}{4} \Omega\left(h_{0}\right)=\frac{\pi}{2} \sin (\pi t)
$$

where we have integrated $\tilde{\Theta}(h)$ to get

$$
\Omega\left(h_{0}\right)=\int_{0}^{1} \sin (\alpha \pi(t+s-1)) h_{0}(t+2 s, \tau)+\sin (\alpha \pi(t-s+1)) h_{0}(t-2 s, \tau) d s .
$$

Naturally the solution to this equation is subject to the prescribed periodic boundary condition,

$$
h_{0}(t, \tau)=h_{0}(t-2, \tau)
$$

as well as the initial condition,

$$
h_{0}(t, 0)=0 .
$$

The choice of the name for (2.39) is fairly straightforward. We first note that nomenclature from the theory of integral equations stipulates that any equation in which the dependent variable appears in a integral whose limits are constants is called a Fredholm integral equation [6]. As the limits of the integral term are constants in our case, and taking $\beta=0$ recovers the forced KdV equation, we name (2.39) the forced KdV-Fredholm equation.

One can notice immediately that the result of Cox and Mortell [12] is recovered when the bottom forcing is neglected $(\beta=0)$ giving the forced Korteweg and De-Vries equation studied by Amundsen et al. [3]. However, the presence and the form of the function $\Omega\left(h_{0}\right)$ is novel and its treatment constitutes the focus of this thesis. The final 
condition $h_{0}$ must satisfy, crucial to studying the steady state, is a zero mean over a period of 2 in the fast time. This amounts to conservation of fluid in the tank. To determine whether the KdV-Fredholm equation satisfies this property, we will need more information about $\Omega\left(h_{0}\right)$ discussed in the following subsection.

\subsubsection{Influence of Vertical Forcing and The Mean Condition}

A quick examination of $\Omega$ shows it is a nonlinear average of the bottom forcing and the velocity potential over the spatial range of $x$. Furthermore, the PDE obtained is a representation of the $x$ velocity component at the mobile boundary, so the $\Omega$ term can be seen as the nonlinear average of the vertical forcing and the potential that affects the velocity component perpendicular to the motion of bottom forcing. This is the type of coupling we were seeking when choosing scalings. One rather surprising result is that $\Omega\left(h_{0}\right)=0$ for any odd $\alpha$. This is not difficult to show but involves some rearrangements. We first denote the forward and backward travelling portion of the term $I_{1}$ and $I_{2}$ as follows,

$\Omega^{(o)}\left(h_{0}\right)=\overbrace{\int_{0}^{1} \sin \alpha^{(o)} \pi(t+s-1) h_{0}(t+2 s, \tau) d s}^{I_{1}}+\underbrace{\int_{0}^{1} \sin \alpha^{(o)} \pi(t-s+1) h_{0}(t-2 s, \tau) d s}_{I_{2}}$,

where the superscript of $o$ is in place to denote odd. What is immediately noticeable is that, by the oddness of $\alpha^{(o)}, I_{1}$ can be written,

$$
I_{1}=-\int_{0}^{1} \sin \alpha^{(o)} \pi(t+s) h_{0}(t+2 s, \tau) d s,
$$

and similarly for $I_{2}$. Substituting $\tilde{s}=-s$ for $I_{2}$ and interchanging the limits of integration gives

$$
I_{2}=-\int_{-1}^{0} \sin \alpha^{(o)} \pi(t+\tilde{s}) h_{0}(t+2 \tilde{s}, \tau) d \tilde{s} .
$$

Shifting by $s_{1}=\tilde{s}+1$, using the oddness of $\alpha$ once again and applying the periodicity of $h_{0}$ and leaves,

$$
I_{2}=-\int_{0}^{1} \sin \alpha^{(o)} \pi\left(t+s_{1}-1\right) h_{0}\left(t+2 s_{1}-2, \tau\right) d s_{1}=-I_{1},
$$


giving $\Omega^{(o)}\left(h_{0}\right)$ is equal to zero. Similarly, it can be shown that, for any even integer $\alpha^{(e)}$,

$$
\Omega^{(e)}=2 \int_{0}^{1} \sin \alpha^{(e)} \pi(t+s) h_{0}(t+2 s, \tau) d s .
$$

So in the case of odd integer $\alpha$, the bottom forcing, including a forcing at the fundamental frequency, has no identifiable nonlinear influence on the $x$ velocity of the wave at this order. This is counter-intuitive and likely indicates that the nonlinear effects of odd integer forcing are encapsulated in a higher order nonlinearity, for example $h_{0}^{2} \frac{\partial h_{0}}{\partial t}$ rather than within an integral term and their representation would require different scalings. As even integers are those giving rise to new phenomena, we examine these exclusively herein.

To derive the mean condition, we need to make a few further re-arrangements to $\Omega$. Assuming $h_{0}$ is a smooth, periodic function, there are a few characteristics of its Fourier series we can exploit. Defining the Fourier series of $h_{0}$ on the general interval $[a, a+2]$ in $t$ by

$$
h_{0}(t, \tau)=\sum_{n} \hat{h}_{0}(n, \tau) e^{\frac{2 \pi i n t}{2}}
$$

then the Fourier coefficients, or modes are given by

$$
\hat{h}_{0}(n)=\frac{1}{2} \int_{a}^{a+2} h_{0}(t, \tau) e^{-2 \pi i \frac{n}{2} t} d t
$$

The remarkable property of this representation of $h_{0}$ is that $a$ is arbitrary, i.e. there is no difference between computing a Fourier coefficient on the interval $[t, t+2]$ or $[0,2]$ (see [35]). With this tool in hand, we let $v=t+2 s$ in (2.44), write $\alpha^{(e)}=2 n$ where $n=1,2,3 \ldots$ and use the complex representation of sine to get

$$
\Omega\left(h_{0}\right)=\frac{1}{i} e^{i n \pi t} \int_{t}^{t+2} h_{0}(v, \tau) e^{i n \pi v} d v-\frac{1}{i} e^{-i n \pi t} \int_{t}^{t+2} h_{0}(v, \tau) e^{-i n \pi v} d v
$$

Using the definition of the Fourier modes above this becomes

$$
\Omega\left(h_{0}\right)=\frac{2}{i}\left(\hat{h}_{0}(-n, \tau) e^{i n \pi t}-\hat{h}_{0}(n, \tau) e^{-i n \pi t}\right),
$$

which has the nice property that, for any choice of $n$,

$$
\int_{0}^{2} \Omega\left(h_{0}\right) d t=0
$$


Therefore we can integrate (2.39) over a period $t$, use the periodicity of $h_{0}$ and its initial condition (2.42) to get the final mean condition

$$
\int_{0}^{2} h_{0}(t, \tau) d t=0
$$

rigorously verifying conservation of the fluid volume.

\subsection{Dissipative Theory}

While the invisicid approximation to studying waves has a long history of being historically successful, the reality is that any fluid system has some dissipative viscous effects [2]. The theory of dissipation in fluid systems such as this is really an area of research in and of itself, for instance see [13]. Chester [9] analyzed the case in which dissipation arose due to a Stokes boundary layer along the walls of the tank giving rise to the additional convolution term

$$
\frac{1}{4} \zeta\left\{\int_{\infty}^{\infty}(\operatorname{sgn}(r)+1) \frac{\partial h_{0}}{\partial t}(t-r, \tau)|\pi r|^{\frac{-1}{2}} d r\right\} .
$$

If incorporated, this would be appear on the left side of (2.39) and we note that $\zeta$ is proportional to the square root of the kinematic viscosity of the fluid. The main argument to simplify this term and place it in an analytically tractable framework is that the kinematic viscosity is also a small parameter. So small in fact, that there are little distinguishing attributes differentiating the above integral term and a linear damping term. This is the argument of Cox and Mortell [12] and it gave excellent agreement with the forced KdV with the Stokes boundary layer damping term. This choice does, however, come with the undesired consequence of affecting the mean condition. Therefore, in this work we adopt a weak Burgers viscosity term, which also has been shown to have similar physical attributes in Amundsen et al. [3] making (2.39)

$$
\frac{\partial h_{0}}{\partial \tau}+\frac{3}{2} h_{0} \frac{\partial h_{0}}{\partial t}+\Delta \frac{\partial h_{o}}{\partial t}-\frac{\kappa}{6} \frac{\partial^{3} h_{0}}{\partial t^{3}}-\frac{\tilde{\beta} \alpha \pi}{4} \Omega\left(h_{0}\right)-\mu \frac{\partial^{2} h_{0}}{\partial t^{2}}=\frac{\pi}{2} \sin (\pi t),
$$

where $\mu \ll 1$ but is $\mathcal{O}(1)$ in $\varepsilon$. One can readily verify that the inclusion of such a term does not affect the mean condition, thus, from a conservation perspective this choice is consistent with the basic physics of the system. We will call (2.50) the forced KdVFredholm-Burgers equation of fKdVFB for short. 
It should be mentioned, the inclusion of this term is a significant simplification of the dissipation in more violent instances of sloshing. Faltinsen and Timokha [13] considered sloshing systems in which the tank was pitched and surged as described in the introduction. These systems can lead to substantially more complex wave behaviour, specifically "run-up" and breaking of the waves. Run-up is simply the wave travelling so far up one of the walls that the normal of the surface nearest to the wall becomes horizontal. After this occurs, there is a significant energy loss from the wave imploding on itself [13]. It is this same implosion which gives rise to the energy loss from waves breaking [13]. Systems in which this kind of behaviour is possible pose a significant challenge numerically, waves breaking only being properly predicted computationally by Grilli et al. in 1993 [19]. Needless to say, systems such as this pose a mathematically daunting challenge analytically [26]. In light of this, we accept the simplification embedded in Burgers damping term to continue the pursuit of an analytically tractable model of sloshing. The compromise is that, if such violent behaviour akin to the observations in Faltinsen and Timokha [13] were to arise in a (for now, hypothetical) experiment of our system, this model would likely not be able to predict it.

\subsection{Summary}

- A periodically forced $\mathrm{KdV}$-Fredholm equation subject to a zero mean condition and periodic boundary condition was shown to be the governing equation for an inviscid fluid being resonantly forced from the bottom and side of a open container.

- It was shown that, if the frequency of the bottom forcing is an odd multiple of the side forcing, the governing equation is simply the periodically forced KdV equation.

- Burgers damping was incorporated into the forced KdV-Fredholm equation to capture the physically present dissipation effects. 


\section{Chapter 3}

\section{The Steady-State Forced KdV}

\subsection{Introduction}

Having our model equation for nonlinear sloshing, the remaining chapters are devoted to analyzing it perturbatively. To clean up the fKdVFB equation(2.39), we allow for a small abuse of notation, letting

$$
\tilde{x}=t, \quad U(\tilde{x}, \tau)=h_{0}(t, \tau), \quad \lambda=\frac{\kappa}{6}, \quad \nu=\frac{\tilde{\beta} \alpha \pi^{2}}{2}, \quad \alpha_{e}=\alpha,
$$

and drop the tilde's to consider the equation, without loss of generality, in the domain $x=[-1,1]$ and $t=[0, \infty)$

$$
U_{\tau}-\lambda U_{x x x}+\frac{3}{2} U U_{x}+\Delta U_{x}-\frac{2 \nu}{\pi} \int_{0}^{1} \sin \alpha \pi(x+s) U(x+2 s, \tau) d s-\mu U_{x x}=\pi \sin (\pi x) .
$$

We have also dropped the factor of one-half previously acting on the horizontal forcing which amounts to a small re-scaling, $l \rightarrow 2 l$, at the beginning of the derivation. Staying in line with the physics of the problem, we search for a function $U(x, \tau)$ which is 2-periodic in $x$ and satisfies the mean condition,

$$
\int_{-1}^{1} U(x, \tau) d x=0
$$

For the remainder of the study we only consider the case $\alpha=2$, however, it should be stressed that the ensuing methodology applies to any choice of even alpha. In this case the fKdVFB is given by,

$$
U_{\tau}-\lambda U_{x x x}+\frac{3}{2} U U_{x}+\Delta U_{x}-\frac{2 \nu}{\pi} \int_{0}^{1} \sin 2 \pi(x+s) U(x+2 s) d s-\mu U_{x x}=\pi \sin (\pi x) .
$$


While this PDE-integral equation presents an interesting study in its own right, we will be concerned with the steady-state implying $U_{\tau}(x, \tau)=0$ and necessarily $U(x, \tau)=U(x)$ leaving the integro-differential equation,

$$
\lambda U_{x x x}=\frac{3}{2} U U_{x}+\Delta U_{x}-\frac{2 \nu}{\pi} \int_{0}^{1} \sin 2 \pi(x+s) U(x+2 s) d s+\mu U_{x x}-\pi \sin (\pi x) .
$$

Using a linear transformation $U(x)=u(x)-\frac{2}{3} \Delta$ this can be reduced to

$$
\lambda u_{x x x}=\frac{3}{2} u u_{x}-\frac{2 \nu}{\pi} \int_{0}^{1} \sin 2 \pi(x+s) u(x+2 s) d s+\mu u_{x x}-\pi \sin (\pi x) .
$$

The mean condition for $u(x)$ then becomes,

$$
\int_{-1}^{1} u(x) d x=\frac{4}{3} \Delta
$$

Adopting the complex representation of sine and using the definition of the Fourier modes from (2.45), we can rewrite the $\int_{0}^{1} \sin 2 \pi(x+s) u(x+2 s) d s$ term so that the above becomes

$$
\lambda u_{x x x}=\frac{3}{2} u u_{x}-\frac{2 \nu}{\pi i}\left(\hat{u}(-1) e^{i \pi x}-\hat{u}(1) e^{-i \pi x}\right)+\mu u_{x x}-\pi \sin (\pi x) .
$$

We are now able to integrate once in $x$ to yield,

$$
\lambda u^{\prime \prime}(x)=\frac{3}{4} u^{2}(x)+\nu\left(\hat{u}(-1) e^{i \pi x}+\hat{u}(1) e^{-i \pi x}\right)+\mu u^{\prime}+\cos (\pi x)-c .
$$

where $c$ is a constant of integration. To complete the clean-up, we replace $\left(\hat{u}(-1) e^{i \pi x}+\right.$ $\left.\hat{u}(1) e^{-i \pi x}\right)$ with the integral term $\int_{-1}^{1} \cos \pi(x+v) u(v) d s$ to get the principal ordinary integro-differential equation of interest

$$
\lambda u^{\prime \prime}(x)=\frac{3}{4} u^{2}(x)+\nu \int_{-1}^{1} \cos \pi(x+v) u(v) d v+\mu u^{\prime}+\cos (\pi x)-c .
$$

Equation (3.6) is now the centrepiece for of our study.

As our variables have undergone a number of changes and redefinitions, it is worthwhile to explain how the model equation above (3.6) connects with the original physical sloshing problem. The first note is that the variable $x$ in fact represents a time variable and can be viewed as real-time at the steady-state. The dependent variable $u(x)$ represents the non-dimensionalized horizontal velocity component of the fluid at the mobile right side of the tank, or $\phi_{0 x}(x, y, t)$ in original variables. Thus by plotting approximate solutions $u(x)$ versus $x$ we are plotting the non-dimensionalized horizontal velocity of 


\begin{tabular}{|c|c|c|}
\hline Parameter & Physical Meaning & Original Variables \\
\hline \hline$\lambda$ & Aspect ratio to side forcing. & $\delta^{2} / \varepsilon^{\frac{1}{2}} 6$ \\
\hline$\nu$ & Relative strength of bottom forcing to side. & $\mathcal{O}\left(\gamma / \delta^{2} \varepsilon^{\frac{1}{2}}\right)$ \\
\hline$\Delta$ & Difference of forcing frequency from the resonant. & - \\
\hline$\mu$ & Strength of viscous effects. & - \\
\hline
\end{tabular}

Table 3.1: Physical characteristics of the coefficients in the steady-state KdV-Fredholm (3.6).

the fluid at the side of the tank in real time, over the domain of one period. The relation between the velocity potential and the free surface is then given by (2.21), or the free surface condition in which $\eta_{0}$ is eliminated from the derivation. Direct comparison with experiment or computational studies is possible by simply re-dimensionalizing the velocity potential, $u(x)$, and the time variable, $x$ and using the relationship with the free surface given in equation (2.21). The physical interpretations of the remaining constant parameters in the equation are also somewhat masked by a series of renaming in the derivation and clean-up. Their physical description as well as explicit relations to the original variables is given in Table 3.1. The parameter $\nu$ has undergone a number of renamings so its order in original variables is given rather than its explicit expression.

As mentioned previously, the novelty of equation (3.6) rests solely in the presence of the Fredholm integral term. As such, we study the equation by increasing its perturbative strength incrementally. This chapter is devoted to the case in which $\nu=0$, which physically corresponds to removing the bottom forcing from the theoretical framework. Chapter Four introduces the $\nu$ as non-zero, but weakly so and Chapter Five considers the case in which $\nu$ is order one. For now, we ignore the damping and return to it in Section 3.6. Taking $\nu=0$ and $\mu=0$ in (3.6) leaves us with the steady-state forced KdV equation

$$
\lambda u^{\prime \prime}(x)=\frac{3}{4} u^{2}(x)+\cos (\pi x)-c .
$$

This equation was studied by Amundsen et al. [3] and thus this chapter amounts largely to a review of their work with a comment on a question arising from their work.

In this chapter we study (3.7) with and without damping. In Section 3.2 we study the phase space of (3.7) when the forcing is constant. In Section 3.3 we outline the asymptotic assumptions to study (3.7) perturbatively and introduce the methodology used to seek its asymptotic solutions. In Section 3.4 we derive the outer, non-dispersive 
solutions to (3.7). In Section 3.5 we derive the inner solutions to (3.7) and in Section 3.6 we illustrate the analytic consequences of introducing dissipation.

\subsection{The Phase Plane}

To study (3.7) qualitatively consider the equation

$$
u^{\prime \prime}(x)=\frac{3}{4} u^{2}(x)+F
$$

where $F$ is a constant such that $F<0$. This equation eventually arises within a boundary layer for small $\lambda$ and $F$ corresponds to the forcing within the layer. Figure 3.1 shows phase plane for the autonomous equation above in the case when $F=-1$. In the subsequent analysis, the saddle point and centre correspond to slowly moving outer solutions of the non-autonomous fKdV equation while the trajectories correspond to the rapidly varying inner solutions, the qualitative behaviour being shown in Figure 3.2. The principal areas of interest for the inner solutions are the regions inside and outside of the homoclinic orbit. The majority of solutions outside of the homoclinic become unbounded, however, there is one trajectory which exits the saddle point, traverses terrain exterior to the homoclinic and then returns to the saddle point where it originated. This type of solution we will call a single orbit exterior solution and is illustrated in Figure $3.2 \mathrm{~b}$ ). The same single orbit possibility exists within the region enclosed by the homoclinic, but more richly, solutions may enter the homoclinic and orbit the centre as many times as the mean condition permits before returning to the saddle point, illustrated on the left of Figure 3.2. It is these multiple orbit solutions that comprise the main interest of this chapter and the single orbit solutions will be discussed later on in Chapter Five.

\subsection{Asymptotic Assumptions and Modulation The- ory}

\subsubsection{Asymptotic Assumptions}

To be able to handle (3.7) perturbatively, a small parameter needs to be decided upon. In the proceeding analysis and subsequent chapters we assume a small dispersion parameter,

$$
\lambda \ll 1 \text {. }
$$




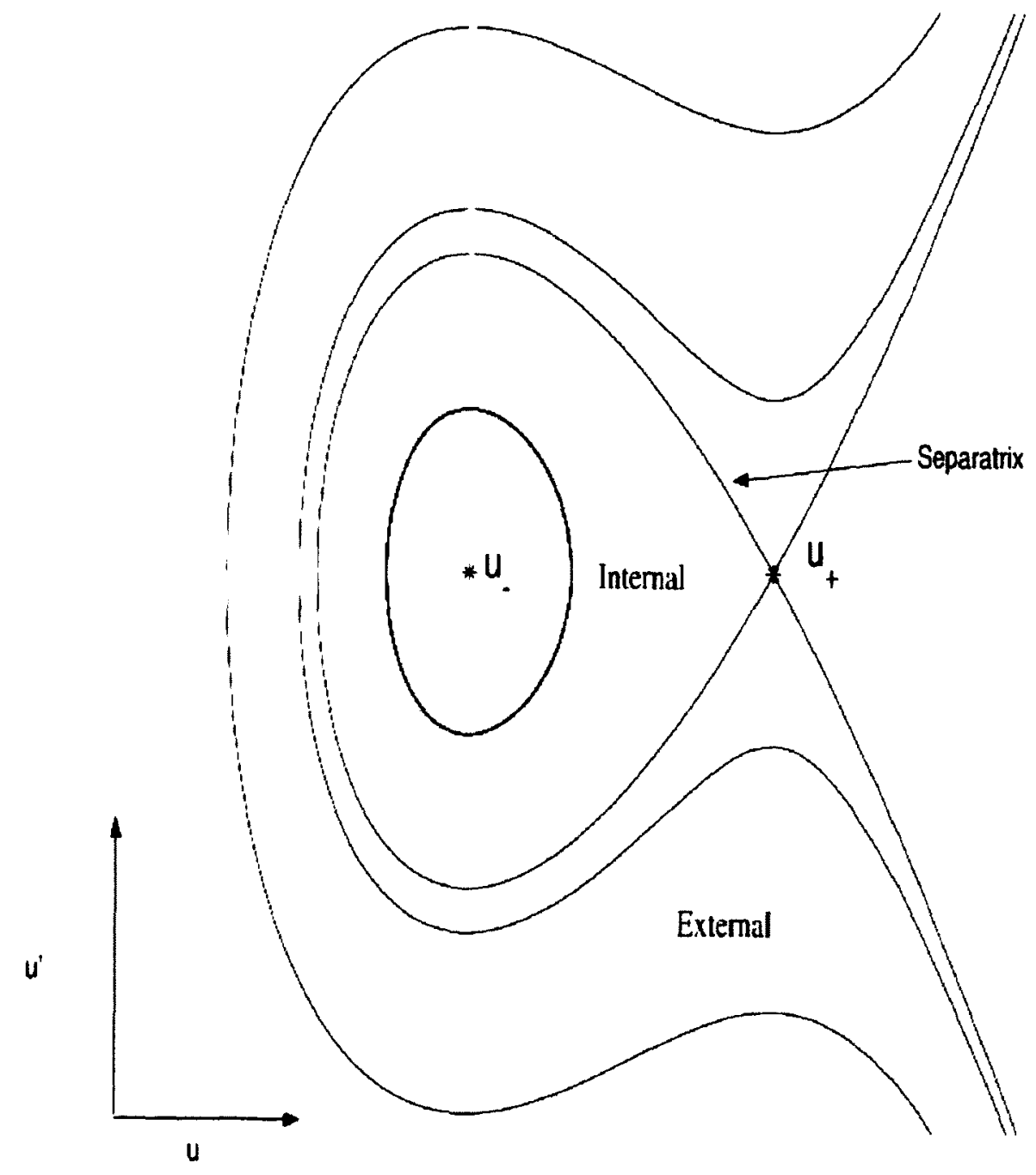

Figure 3.1: Figure 1a) in Amundsen et al. illustrating the phase plane to the autonomous, constant F, system (3.8) [3]. 

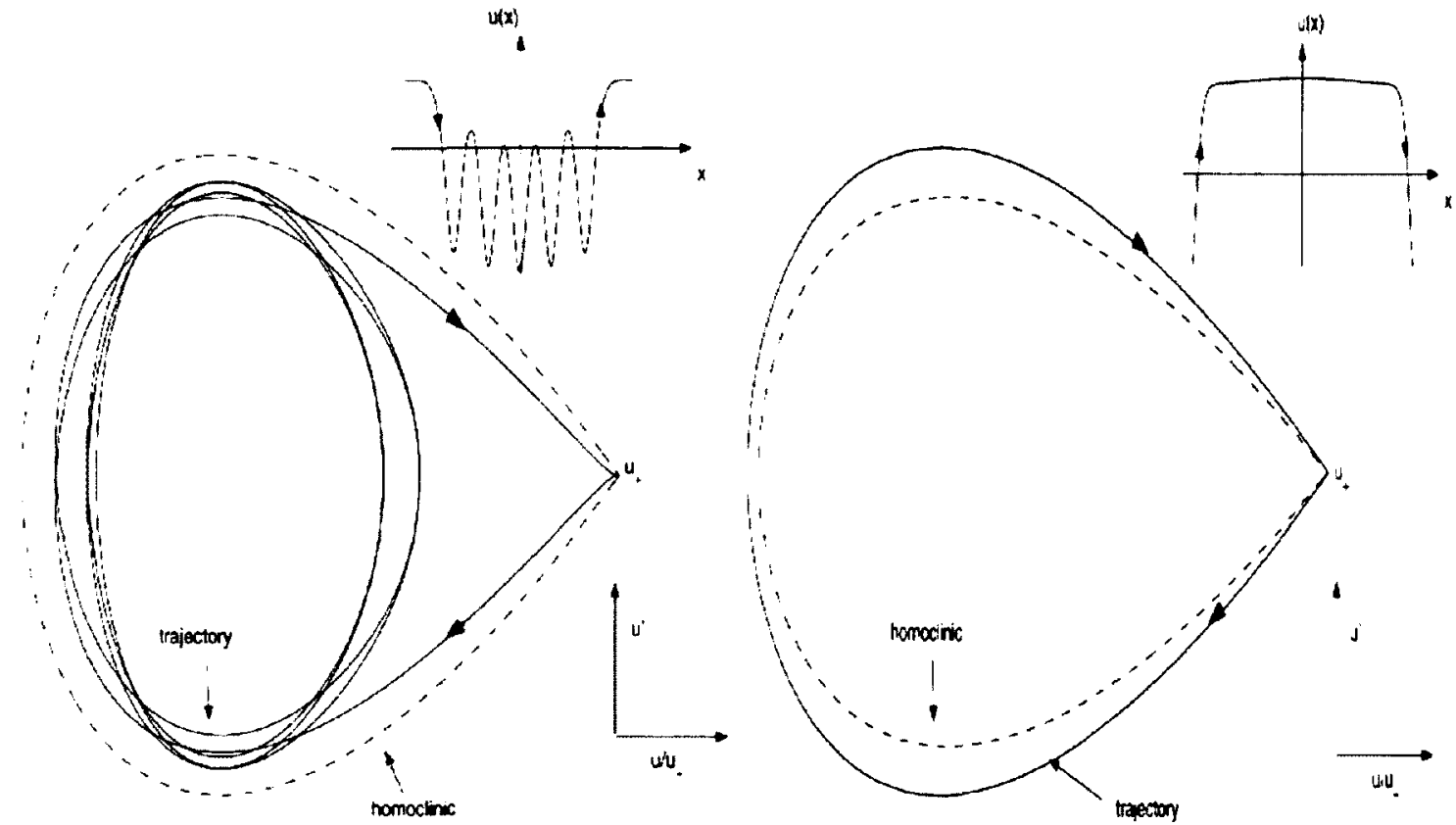

Figure 2. Multiple orbit trajectories for a) internal solution (left). b) external solution (right). Inset is corresponding spatial dependence of solution over one period.

Figure 3.2: Figure $2 \mathrm{a}$ ) and b) in Amundsen et al.[3] illustrating the various qualitative possibilities of the non-autonomous $\mathrm{fKdV}$ system within the boundary layer, ie. (3.8) where $\mathrm{F}$ now varies slowly. The fixed points are now the outer perturbative solutions to (3.7) allowing the trajectories (the rapidly varying inner solutions) to cross one another. 
As a reminder, $\lambda$ measures the ratio of the square of the aspect ratio of the tank to the root of the horizontal forcing see Table 3.1. This small dispersion assumption is largely an experimentally verified assumption. Aside from strong agreement of the work of Amundsen et al. [3] with the experiments of Chester and Bones [3], the paper by Faltinsen and Timokha [13] comments that small dispersion was observed even in systems with particularly violent behaviour.

While there is a feel of a Russian doll type stacking of small parameters to the small dispersion assumption, this is justified by recalling the imposition that $\kappa$ remain order one in $\varepsilon$. Therefore there is nothing impeding us theoretically from taking $\kappa$ to be small provided it remains independent of powers of epsilon. The small dispersion limit places (3.7) within reach of a perturbative technique known as modulation theory which we outline in the next section.

\subsubsection{Modulation Theory}

We now turn our attention to seeking leading order asymptotic solutions to (3.7). To do so we employ the method of Amundsen et al. [3] which can be summarized as follows,

1. Determine the leading order outer ("non-dispersive") solutions by expanding $u(x)$ in a regular perturbation series and solving the leading order problem.

2. Determine the leading order inner ("dispersive") solutions by introducing a generalized multiple scales argument.

3. The dispersive solutions will depend on two free, slowly varying parameters which we determine by removing secularities. The equations arising from removing secularities can be considered the "modulation equations"

The method finds its roots in one slightly narrower in scope developed by nonlinear wave pioneer Gerald Whitham [37, 38]. Whitham's elegant technique involves finding quickly varying, travelling wave solutions to nonlinear wave PDE's in terms of a generalized phase function depending on slowly varying spatial and temporal variables. The leading order solutions to these wave problems is then solved, leaving its parameters to vary slowly. To rigorously determine these parameters, variations in the PDE's Lagrangian in terms of the slowly varying parameters are then averaged. In the case that such a Lagrangian is unavailable, Luke [25] showed that the slowly varying parameters can be found using secularity removal techniques such as the Fredholm Alternative described later in this 
chapter. Luke's work is an extension to partial differential equations of the method developed by Kuzmak [24] for ordinary differential equations and thus, in literature, the method employed by Amundsen et al. is sometimes referred to as the Luke-Kuzmak method [22]. Broadly speaking, all of these methods fall under an area of nonlinear wave research known as "modulation theory". A perturbative technique, the overarching idea in modulation theory is to seek rapid solutions with slowly varying parameters and then determine how these slowly varying parameters are "modulated" either averaging conservation laws [27], averaging Lagrangians [38] or removing secularities [25]. We comment on this to address a question arising from the work of Amundsen et al. regarding a global, nonlinear WKB technique commented on in Trinh [36].

\subsection{Non-dispersive solutions}

To initiate the analysis, we follow Amundsen et al. [3] and seek the non-dispersive solutions by assuming a regular perturbation expansion in $\lambda$

$$
u(x)=\sum_{n=0}^{N} \lambda^{n} y_{n}(x)
$$

giving the leading order, non-dispersive solutions as

$$
y_{0}= \pm \frac{2}{\sqrt{3}} \sqrt{c-\cos (\pi x)}
$$

which are defined and smooth provided $c>1$. What one can see clearly from the nondispersive solutions is that they are of a single sign, which implies there is a fairly limited range of $\Delta$ values of the mean condition (3.4) that these solutions can satisfy. Of course, mathematically a combination of the two non-dispersive solutions connected by a shock is possible, however, in the context of liquid fluids this is an unsatisfactory solution and not one observed in numerical or physical experiments. The limitations of the non-dispersive solutions imply the existence of resonant band, briefly addressed in the derivation, in a neighbourhood of $\Delta=0$ which we delineate by $\Delta_{m}$ and $\Delta_{M}$ corresponding to the minimum and maximum values of $\Delta$ for which the non-dispersive solutions are valid. Integrating (3.9) over the period [18] gives

$$
\int_{-1}^{1} y_{0}(x) d x= \pm \frac{4 \sqrt{3} \sqrt{c+1}}{\pi} E\left(\frac{\sqrt{2}}{c+1}\right) .
$$


Taking the limit as $c \rightarrow 1$, we find from the mean condition (3.4) the resonant band is delineated by

$$
\Delta_{M}=-\Delta_{m}=\frac{2 \sqrt{6}}{\pi}
$$

Now that we have a quantitative grasp of the boundaries of the resonant band, we pursue the rapidly varying dispersive solutions.

\subsection{Dispersive Solutions}

In the analysis of Amundsen et al. [3] there were two types of dispersive solutions considered. The first were single orbits (external and internal), found using boundary layer analysis and the second were multiple internal orbits using the generalized multiple scales. In this section we concern ourselves with the rich possibilities of multiple internal orbits and return to a discussion of the single orbit solutions in Chapter Five. To derive the multiple orbit dispersive solutions, we introduce a generalized multiple scale as discussed in the Section 3.3 defined by

$$
X=\frac{\omega_{1}(x)}{\sqrt{\lambda}}+\omega_{2}(x)+\ldots
$$

and expand $u(x)$ as follows,

$$
u(x)=z_{0}(x, X)+\sqrt{\lambda} z_{1}(x, X)+\ldots
$$

The derivative operator is then transformed to,

$$
\frac{d^{2}}{d x^{2}} \rightarrow\left(\frac{d X}{d x}\right)^{2} \partial_{X}^{2}+2 \frac{d X}{d x} \partial_{x} \partial_{X}+\frac{d^{2} X}{d x^{2}} \partial_{X}+\partial_{x}^{2}
$$

As we are only concerned with deriving a leading order solution, it is the first two orders of the perturbative hierarchy that hold any influence on our analysis. With this in mind, substituting $X$ and collecting powers of $\lambda$ gives the new relevantly truncated operator,

$$
\frac{d^{2}}{d x^{2}} \rightarrow \frac{\left(\omega_{1}^{\prime}\right)^{2}}{\lambda} \partial_{X}^{2}+\frac{1}{\sqrt{\lambda}}\left(2 \omega_{1}^{\prime} \omega_{2}^{\prime} \partial_{X}^{2}+2 \omega_{1}^{\prime} \partial_{x} \partial_{X}+\omega_{1}^{\prime \prime} \partial_{X}\right)+\ldots
$$

Applying this to (3.11) and substituting into (3.7) yields, to leading order,

$$
\left(\omega_{1}^{\prime}\right)^{2} z_{0 X X}=\frac{3}{4} z_{0}^{2}-c+\cos (\pi x)
$$


The above has a solution which can be written in closed form using the Jacobi cnoidal function

$$
z_{0}=d+a \mathrm{cn}^{2}\left(k\left(X-\delta_{1}\right), m\right) .
$$

where $\delta_{1}$ is a phase shift to be determined and the slowly varying parameters are given by

$$
a=-8\left(\omega_{1}^{\prime}\right)^{2} m^{2} k^{2}, \quad d=\frac{8}{3}\left(\omega_{1}^{\prime}\right)^{2} k^{2}\left(2 m^{2}-1\right), \quad k=\left(\frac{3(-c+\cos (\pi x))}{16\left(\omega_{1}^{\prime}\right)^{4}\left(m^{4}-m^{2}+1\right)}\right)^{\frac{1}{4}}
$$

Here we see that there are two slowly varying parameters that at present are undetermined: the derivative of the stretched variable $\omega_{1}^{\prime}$ and the elliptic modulus $m$ which dictates the modulation of frequency in the wave. The next step is to supply the so-called modulation equations which ensure well-orderedness of the series.

\subsubsection{Removing Secularity}

The first measure to avoid secularity is to impose a constant period $T$ in $X$ of the function. In general, for nonlinear wave equations, Luke [25] describes that the rapidly varying solutions are oscillatory ones, periodically visiting a neighbourhood of a nondispersive solutions as illustrated in Figure 3.2 and thus be periodic [25]. The fact that this period must be independent of $x$ requires a more subtle argument. To see the effect a period dependent on $x$ would have, note that for functions $z_{i}(x, X)$ that are $T$-periodic in $X$ we would have the relation for each $i, n \in \mathbb{N}$,

$$
z_{i}(X, x)=z_{i}(X+n T(x), x) .
$$

Taking the full derivative yields

$$
\frac{d X}{d x} \partial_{X} z_{i}=\frac{d X}{d x} \partial_{X} z_{i}(X+n T(x), x)+n \frac{d T}{d x} \partial_{X} z_{i}(X+n T(x), x),
$$

which we can see becomes unbounded for large $n$ and relatedly, for large $X$ (note that this is the argument of Luke [25]). Thus to eventually enforce a match to the non-dispersive solutions we require this period $T$ to be simply a constant. This argument, however, does not specify what constant the period must be. This choice is arbitrary as it amounts to a constant stretching of the fast variable $X$ which can be absorbed by the $\omega_{i}(x)$ functions. 
From the properties of the cnoidal function (see [1]) applying periodicity gives

$$
T=\frac{2 K(m)}{k}=\frac{4 K(m) \omega_{1}^{\prime}\left(m^{4}-m^{2}+1\right)^{\frac{1}{4}}}{3^{\frac{1}{4}}(-c+\cos (\pi x))^{\frac{1}{4}}},
$$

where $m=m(x)$ and $K$ is the complete elliptic integral of the first kind. We now have the first necessary expression for the leading order of the stretched variable in terms of the modulus. Taking $T=\frac{4}{3^{\frac{1}{4}}}$ for convenience yields

$$
\omega_{1}^{\prime}=\frac{\left(-c+\frac{1}{2} \cos (\pi x)\right)^{\frac{1}{4}}}{K(m)\left(m^{4}-m^{2}+1\right)^{\frac{1}{4}}} .
$$

To find a second condition to close the system, we turn to the next order in the perturbative hierarchy given by

$$
\left(\omega_{1}^{\prime}\right)^{2} z_{1 X X}-\frac{3}{2} z_{0} z_{1}=-2 \omega_{1}^{\prime} \omega_{2}^{\prime} z_{0 X X}-2 \omega_{1}^{\prime} z_{0 X X}-\omega_{1}^{\prime \prime} z_{0 X} .
$$

For brevity we write this as

$$
L z_{1}=f(x, X)
$$

where $L$ is given by

$$
L=\left(\omega_{1}^{\prime}\right)^{2} \partial_{X}^{2}-\frac{3}{2} z_{0},
$$

which we recognize as the steady-state Schrödinger operator. The property of $L$ we intend to exploit is self-adjointness. The solution we are seeking is one that is analytic and thus resides in the Hilbert space $C^{\infty}([-1,1] \times(\delta-\sqrt{\lambda}, \delta+\sqrt{\lambda}), \mathbb{R})$, where $[-1,1]$ is the slow variable domain and $(\delta-\sqrt{\lambda}, \delta+\sqrt{\lambda})$ is the fast variable domain, equipped with the standard functional inner product given by,

$$
\langle u, v\rangle=\int_{\mathbb{R}} u(x, X) v(x, X) d X .
$$

For $T$-periodic $u(x, X)$ and $v(x, X)$ we can see that,

$$
\langle L u, v\rangle=\int_{0}^{T}\left(\left(\omega_{1}^{\prime}\right)^{2} u_{X X}-\frac{3}{2} z_{0} u\right) v d X=\int_{0}^{T}\left(\omega_{1}^{\prime}\right)^{2} u v_{X X}-\frac{3}{2} z_{0} u v d X=\left\langle u, L^{*} v\right\rangle .
$$

Obviously $L^{*}=L$ giving

$$
\langle L u, v\rangle=\langle u, L v\rangle .
$$

Thus the operator $L$ acting on any function $u(x, X)$ periodic in $X$ must be orthogonal to homogeneous solutions, ie. if there exists a periodic $\tilde{v}$ a homogenous solution such that $L \tilde{v}=0$ then

$$
\langle L u, \tilde{v}\rangle=0 .
$$


This implies that a necessary and sufficient condition for there to be $T$-periodic solution to $L z_{1}=f(x, X)$ is given a $T$-periodic $\tilde{v}$ such that $L \tilde{v}=0$, then the functional inner product $\left\langle L z_{1}, \tilde{v}\right\rangle=0$, implying for $L z_{1}=f(x, X)$ that

$$
\langle f(x, X), \tilde{v}\rangle=0 .
$$

This is known in analysis as the Fredholm Alternative. What is convenient about taking this step is that we are able to entirely bypass solving for $z_{1}$ to ensure well orderedness of the asymptotic series. What is left is to find such a homogeneous solution. Applying L to $z_{0 X}$ we find

$$
L z_{0 X}=\left(\omega_{1}^{\prime}\right)^{2} z_{0 X X X}-\frac{3}{2} z_{0} z_{0 X}
$$

Differentiating (3.12) with respect to $X$ shows that the left hand side is zero. Having found $z_{0 X}$ to be a homogeneous solution of $L$, we take the inner product of $f(x, X)$ with $z_{0 X}$ to gain our solvability condition

$$
\int_{0}^{T} 2 \omega_{1}^{\prime} \omega_{2}^{\prime} z_{0 X} z_{0 X X}+2 \omega_{1}^{\prime} z_{0 X} z_{0 X X}+\omega_{1}^{\prime \prime} z_{0 X}^{2}=0 .
$$

Noticing that

$$
\frac{d}{d x}\left(\omega_{1}^{\prime} z_{0 X}^{2}\right)=\omega_{1}^{\prime \prime} z_{0 X}^{2}+\omega_{1}^{\prime} \frac{\partial}{\partial X}\left(z_{0 X}^{2}\right)+2 \omega_{1}^{\prime} z_{0 X} z_{0 X x}
$$

implying by periodicity of $z_{0}$ that

$$
\frac{d}{d x} \omega_{1}^{\prime} \int_{0}^{T} z_{0 X}^{2} d X=\int_{0}^{T} \omega_{1}^{\prime \prime} z_{0 X}^{2}+2 \omega_{1}^{\prime} z_{0 X} z_{0 X x} d X
$$

We can then reduce the above solvability condition to

$$
\omega_{1}^{\prime} \overbrace{\int_{0}^{T} z_{0 X}^{2} d X}^{Q}=C_{1}
$$

where $C_{1}$ is a constant. We will find the denotation of $Q$ as delineated in (3.16) to be particularly useful in the next section. Examining the $Q$ term closely, we can see that this is the sum of the kinetic energy within the boundary layer. Thus this type of solvability is a condition enforcing adiabatic invariance [24]. It is the solvability condition that determines the elliptic modulus $m$ on which each of our slowly varying parameters depend on and thus we are finished from a modulation standpoint. One might notice that there are still a few straggler constants that remain undetermined. The first is the phase 
shift $\delta_{1}$, which we take to ensure evenness of the solution. The second is the solvability constant $C_{1}$ which we determine by enforcing that $m \rightarrow 1$ as $X \rightarrow \pm \infty$. This imposition on the solvability constant is what ensures matching between the dispersive and nondispersive layers and depends on the number of orbits the solution takes. Finally, the integration constant $c$ is determined from the mean condition (3.4) for, in principle, a given value of $\Delta$.

\subsubsection{The Resonant Band and Plotting}

The entire resonant response diagram of the fKdV (3.7) is shown in Figure 3.3. The use of the multiple orbit solutions is thus evident. One can construct multiple orbit solutions to satisfy differing values of $\Delta$. In Figure 3.3, solutions with multiple peaks are denoted by $u_{n m}$ where $n$ corresponds to the number of internal orbits and $m$ corresponds to the number of external orbits illustrated in Figure 3.2. The possible values of $\Delta$ a given multiple orbit solution can satisfy is then given by the "finger" next to which the specific $u_{n m}$ is placed. In our work, these are equivalent to the differing number of peaks possible with the rapidly varying $z_{0}(x, X)$ function.

Having constructed the family of solutions across the entire resonant band, we comment that, methodologically, there is something interesting that arises out of Amundsen et al. [3] which one might not expect. As $m \rightarrow 1$, the stretched variable approaches a constant and the multiple scales argument breaks down, see equation (3.15). As such, a matching solution is needed to connect the dispersive and non-dispersive layers to maintain smoothness of the asymptotic solution. This is taken to the be the soliton solution corresponding to the limit as modulus $m(x)$ of the cnoidal solution in (3.13) approaches unity. Figure 3.4 is an illustration from Amundsen et al. showing the matching process between the dispersive and non-dispersive layers.

For reproducibility purposes, Figure 3.4 also helps to explain the interesting difficulty on how a solution plot, rather than just its norm, was obtained later on in Chapter Four and Chapter Five. After finding $m(x)$ and $\omega_{1}$ from their respective nonlinear equations (3.16) and (3.15), the cnoidal or dispersive solution can be plotted readily using the solution expression (3.13). The plotting of the non-dispersive solution is trivial. In general, the matching point where the dispersive regime and non-dispersive regime overlap, $\tilde{x}$, must be found to limit the number of cnoidal peaks to the amount of interest, for instance in later chapters we choose to plot two peaks to illustrate the values of $\Delta$ possible. Once this is done, the matching soliton solution can be used to connect the 


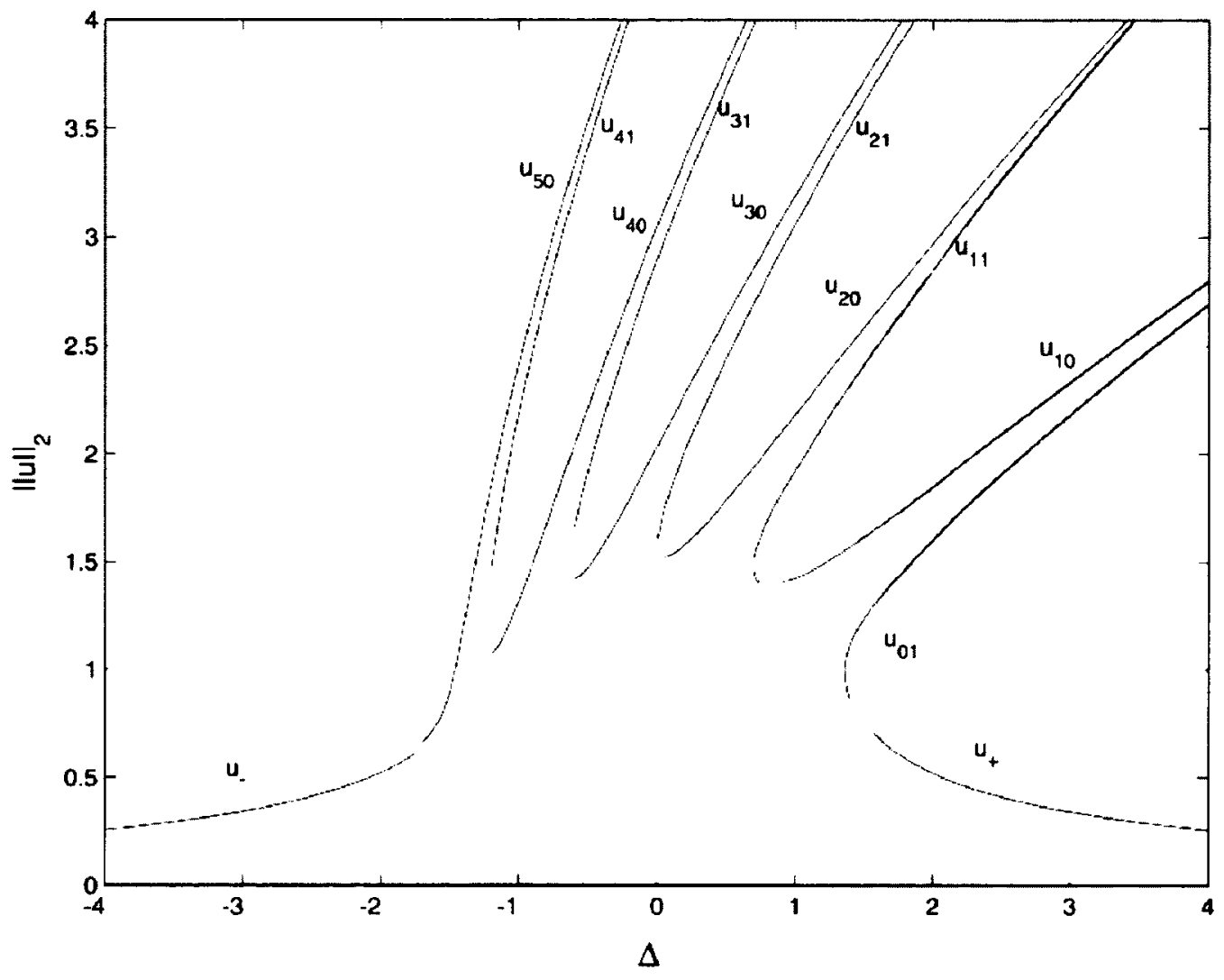

Figure 3.3: Figure 4 from Amundsen et al. [3] illustrating the norm of the inner solutions (in the diagram $u(x)$ in our work $z_{0}(x, X)$ for differing values of $\Delta$. Note the nonuniqueness of solutions for a given value of $\Delta$. 


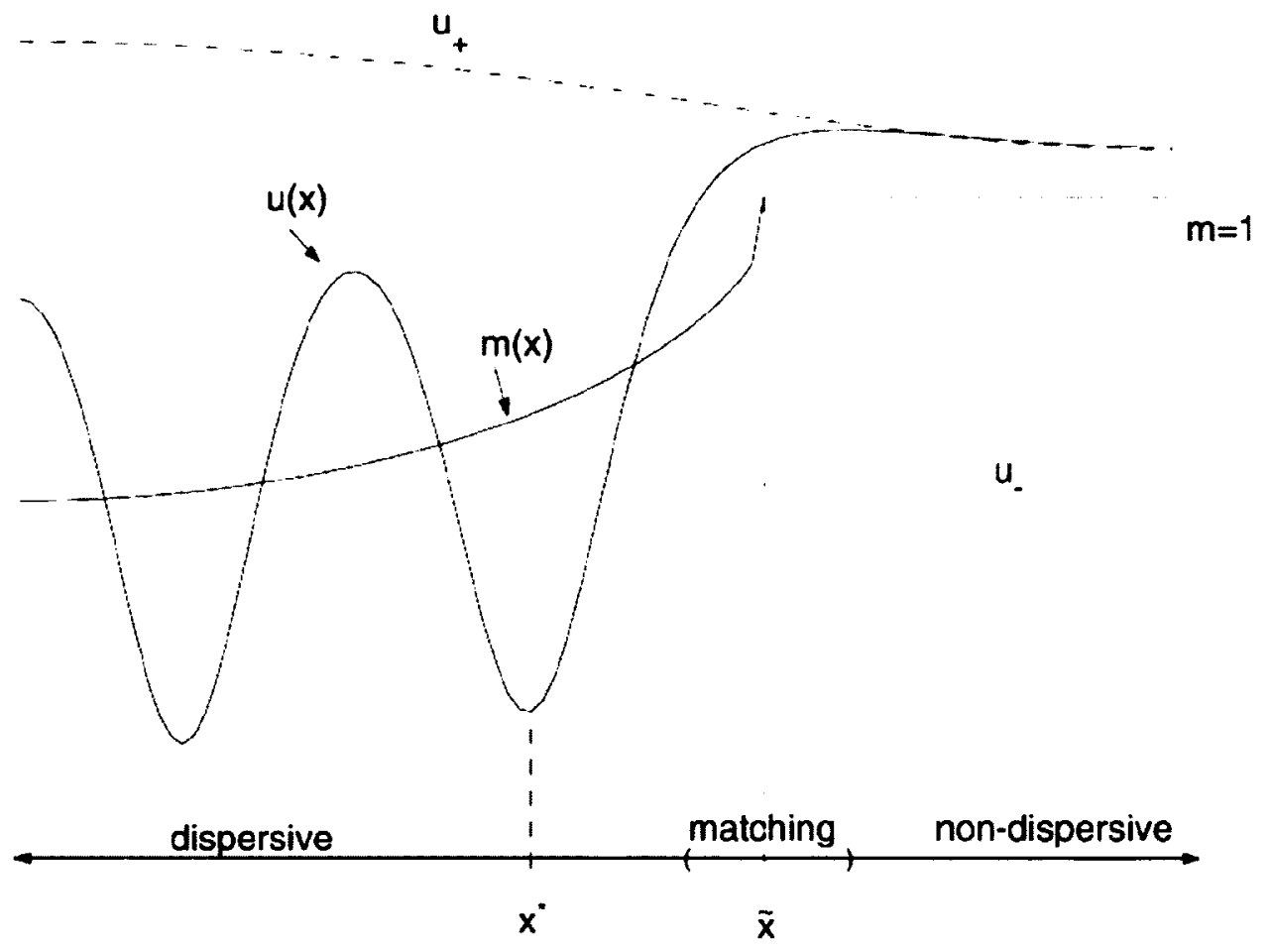

Figure 3.4: Figure 3 from Amundsen et al. [3] illustrating the matching layer between the dispersive cnoidal solutions and non-dispersive solutions. To compare, we write the $u(x)$ in this figure as $z_{0}$ and $u_{ \pm}$as $y_{ \pm}$. A soliton solution, centred at $x^{*}$ is needed to match the cnoidal solutions to the non-dispersive solutions smoothly at the matching point $\tilde{x}$.

two regimes smoothly, the centre of which is taken to be approximately at trough of the final cnoidal solution denoted by $x^{*}$. In general, however, a smooth match is difficult to produce and is largely done by trial and error variation of the matching point $\tilde{x}$. As a result there may be small discontinuities arising from the asymptotic solution which are unavoidable and affect comparison with numerical results presented in Chapter Four and Chapter Five.

The necessity of a matching layer between the dispersive and non-dispersive regimes suggests that the limitation of this multiple-scales technique is its layer driven mentality. Is there a more global technique available? Is there a direct analogue of WKB theory to the nonlinear case? We comment on this question in the next subsection. 


\subsubsection{Nonlinear WKB Comment}

The great success of modulation theory (for example $[16,29]$ ) has lead some to indicate the technique is the nonlinear analogue of the celebrated WKB expansion [21]. Other papers outline a nonlinear WKB method (see Miura and Kruskal [27]) which is effectively equivalent to modulation theory. One can see why this connection is drawn. The WKB expansion [7],

$$
u(x)=\exp \left(\frac{1}{\delta} \sum_{n=0}^{N} \varepsilon^{n} S_{n}\right)
$$

is effective as rapidly varying information can be collected over the entire domain. This collection property owes its entire claim to the fact the series is contained in the exponential which can be factored out of linear differential equations. In the nonlinear case, the definition of the stretched variable contained in the expansion for $u(x)$ has the same information collecting potential but is limited by the function it is contained within. At present, it is widely accepted that there is no one, single function which can be factored out of an arbitrary nonlinear differential equation in the same manner that the exponential can from a linear differential equation. Thus we are forced to put the stretched variable into a general function $z_{0}$. This is perfectly reasonable, we have a stretched variable similar to the expansion within the argument of the exponential and an arbitrary function to be determined from the nonlinear differential equation. The removal of the secularity can then be seen as the equivalent of seeking subdominant functions $S_{i}(x)$.

Then, from the perspective of the nonlinear waves community, modulation theory is already the nonlinear analogue of WKB theory. Why then the multiple-scale break-down in the analysis of Amundsen et al.[3]? Or more importantly, is this a break-down? From a method perspective, modulation theory can only carry the analysis as far as a system of nonlinear equations or first order hyperbolic systems in the case of PDE's. In the fKdV case, the eventual constant nature of the fast variable (see (3.15) as $m \rightarrow 1$ ) is a consequence of the nonlinear system determined for $m(x)$ from the solvability condition. In fact, one can see from the definition of $k(x)$ that forcing the fast variable to be a constant takes $X \rightarrow \pm \infty$. This implies that the method does in fact work and a match is made. The issue is it does not do so smoothly. The parameter $m(x)$ approaches one very rapidly and so does $X$. So rapidly in fact it causes a discontinuity in the matching process and thus the soliton matching solution is an appropriate bridge. Is this really a failure then? The multiple scales technique does work up to the matching process. If we are to draw a connection to WKB theory, this is analogous to a turning point problem, 


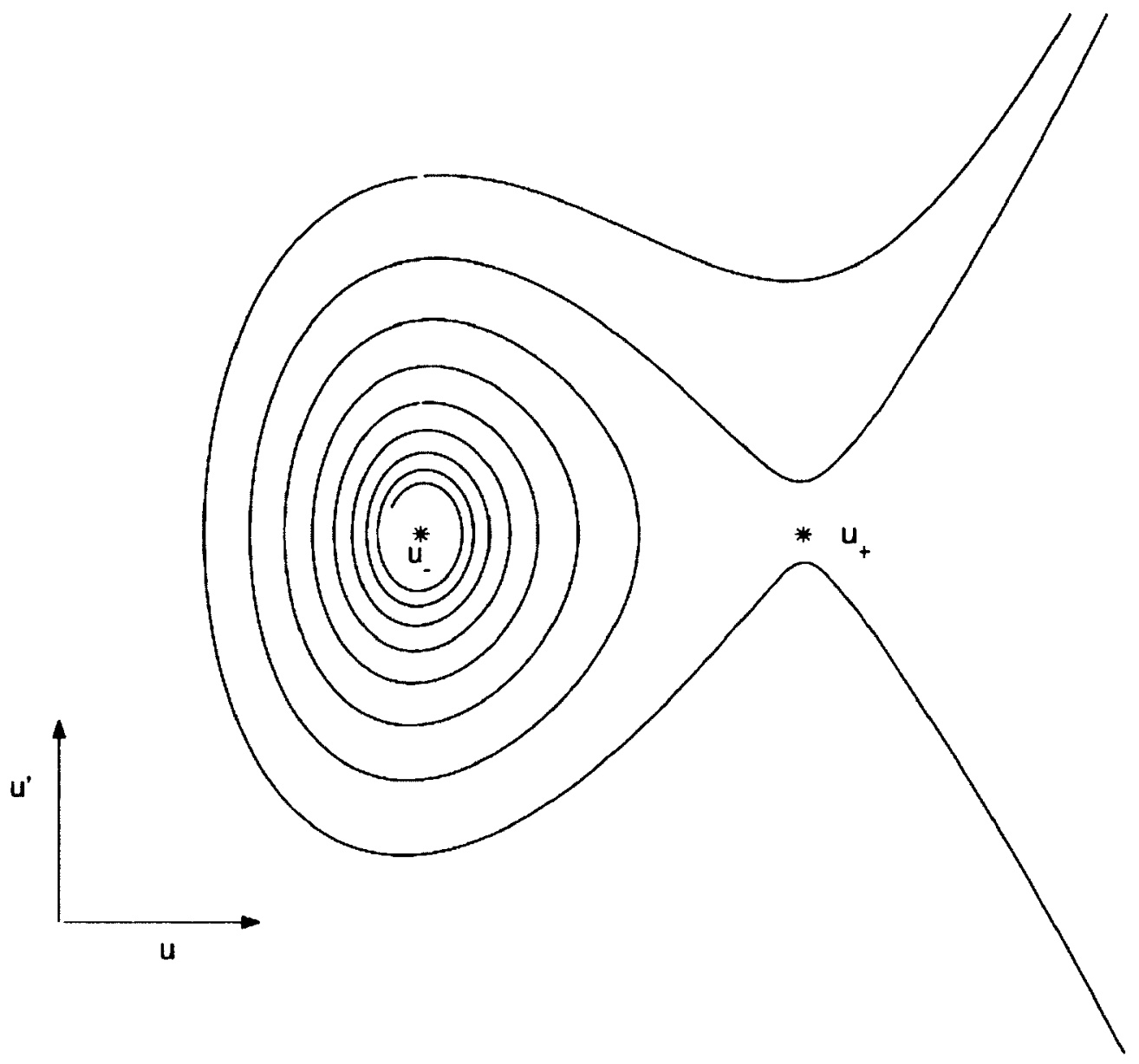

Figure 3.5: Figure 6 from Amundsen et al. [3]. Notice that closed orbits no longer exist

the issue is that we do not have the Airy functions. The soliton connection solution, however, is a boundary layer solution at the turning point where $m(x)=1$ and the fast variable ceases to vary and is thus the appropriate analogue of the Airy functions in this case. We then finish this comment by concluding that modulation theory is a global technique commented on by Trinh [36].

\subsection{Effects of Dissipation}

We now return to (3.6) now with the assumption that $\mu \neq 0$ and $\nu=0$. The phase plane is illustrated for constant forcing and $\mu=0.1$ in Figure 3.5 and we note that closed internal orbits no longer exist. 
As argued in the derivation, the damping is needed to be sufficiently small in order to justify choosing Burgers damping over the Stokes boundary layer integral term. For our purposes, we consider this smallness to be on par with the dispersion and thus take $\mu=\mathcal{O}(\lambda)$. Following the same analysis as the previous Section, introducing the multiple scales yields the same leading order equation and thus the same leading order solution. Periodicity can also be likewise applied, the only difference comes at the second order of the perturbative hierarchy where the damping appears as a potentially secular term. The second order of the perturbative hierarchy is now given by

$$
\left(\omega_{1}^{\prime}\right)^{2} z_{1 X X}-\frac{3}{2} z_{0} z_{1}=-2 \omega_{1}^{\prime} \omega_{2}^{\prime} z_{0 X X}-2 \omega_{1}^{\prime} z_{0 X x}-\omega_{1}^{\prime \prime} z_{0 X}-\omega_{1}^{\prime} \frac{\mu}{\sqrt{\lambda}} z_{0 X} .
$$

Here we use the definition of $Q$ in (3.16) and apply the Fredholm Alternative as before giving

$$
\frac{d}{d x} Q+\frac{\mu}{\sqrt{\lambda}} Q=0
$$

which can be solved to get the damped solvability condition,

$$
\omega_{1}^{\prime} \int_{0}^{T} z_{0 X}^{2} d X=C_{2} e^{-\frac{\mu}{\sqrt{\lambda}} x}
$$

Thus immediately we can notice the impact of the damping, which serves to eliminate unbounded internal solutions to the fKdV equation. Amundsen et al. [3] also comment that there is a stretching of the boundary layer associated with the presence of damping. We however, omit these details. In general, the solutions derived in this chapter agree remarkably with numerical simulations and experiments performed by Chester and Bones [10]. An illustration of the comparison of this technique with numerical results is not included and the interested reader is referred to Amundsen et al. [3]. To continue to push the theory of sloshing forward in an analytic manner, we introduce the integral term in (3.6) with a new strength in the next chapter.

\subsection{Summary}

- We reviewed the technique of Amundsen et al. [3] used in studying the steady-state forced $\mathrm{KdV}$ equation.

- We commented on an open question posed by Amundsen et al. [3] regarding a nonlinear WKB analogue. 


\section{Chapter 4}

\section{The Weak KdV Fredholm Equation}

\subsection{Introduction}

Having the built up the relevant mathematical machinery, we now loosen the restriction of $\nu=0$ in (3.6) and study the equation,

$$
\lambda u^{\prime \prime}(x)=\frac{3}{4} u^{2}(x)+\nu \int_{-1}^{1} \cos \pi(x+v) u(v) d v+\cos (\pi x)-c,
$$

under the same small dispersion assumption that $\lambda \ll 1$ and subject to $u(x)=u(x-2)$ as well as the mean condition,

$$
\int_{-1}^{1} u(x) d x=\frac{4}{3} \Delta
$$

Recall from the previous chapter, this equation can be recast into (3.5) reproduced here,

$$
\lambda u^{\prime \prime}(x)=\frac{3}{4} u^{2}(x)+\nu\left(\hat{u}(-1) e^{i \pi x}+\hat{u}(1) e^{-i \pi x}\right)+\cos (\pi x)-c .
$$

using the properties of Fourier modes mentioned in Chapter Two. We will refer to (4.2) as the "Fourier representation". The insight that (4.1) can be re-cast into the Fourier representation is useful as it illustrates that the integral term corresponds to a secondary forcing, the amplitude of which is dictated by the product of the Fourier modes and the parameter $\nu$. We may then conjecture that, qualitatively, there is little difference between this forced $\mathrm{KdV}$-Fredholm and the forced $\mathrm{KdV}$, including the non-uniqueness of solutions. The question we consider is what can be done analytically with the integral term present. As a first foray into equations of this type, we begin by assuming that 
$\nu=\sqrt{\lambda}$ corresponding to a weak bottom forcing relative to the side forcing. This is a reasonable first line of inquiry to gain some insight into the influence and the properties of the KdV-Fredholm equation. For weak integral terms, the multiple scales argument of Amundsen et al. [3] is well equipped to handle such a problem. We will find at the end of this chapter and its successor that the end result of the asymptotic analysis does is not sufficiently distinct from the undamped case of Amundsen et al. [3] to warrant a detailed study of the damped case and thus its details are not considered.

In this chapter we study (4.1) in the weak bottom forcing regime corresponding to $\nu=O(\sqrt{\lambda})$. In Section 4.2 we derive the non-dispersive and dispersive solutions to (4.1). As will be seen, removing secularity from the dispersive is a problem in its own right and is studied in Section 4.3 and finally in Section 4.4 we compare this work to numerical simulations.

\subsection{Non-dispersive and Dispersive Solutions}

To find the non-dispersive solutions we assume a regular perturbative expansion as before,

$$
u(x)=\sum_{n=0}^{N} \lambda^{\frac{n}{2}} y_{n}(x)
$$

giving to leading order the same non-dispersive solutions as in the previous section,

$$
y_{ \pm}= \pm \frac{2}{\sqrt{3}} \sqrt{c-\cos (\pi x)}
$$

which again are well-defined and smooth provided $c>1$. The resonant band then remains unchanged as well.

Following the analysis in the previous section, we introduce a generalized multiple scale formulation by defining

$$
X=\frac{\omega_{1}(x)}{\sqrt{\lambda}}+\omega_{2}(x)+\ldots
$$

and expanding $u(x)$ by

$$
u(x)=z_{0}(x, X)+\sqrt{\lambda} z_{1}(x, X)+\ldots
$$

The relevant truncated derivative operator remains the same and is given by,

$$
\frac{d^{2}}{d x^{2}} \rightarrow \frac{\left(\omega_{1}^{\prime}\right)^{2}}{\lambda} \partial_{X}^{2}+\frac{1}{\sqrt{\lambda}}\left(2 \omega_{1}^{\prime} \omega_{2}^{\prime} \partial_{X}^{2}+2 \omega_{1}^{\prime} \partial_{x} \partial_{X}+\omega_{1}^{\prime \prime} \partial_{X}\right)+\ldots
$$


Applying this to (4.5) and substituting into (4.1) yields, to leading order,

$$
\left(\omega_{1}^{\prime}\right)^{2} z_{0 X X}=\frac{3}{4} z_{0}^{2}-c+\cos (\pi x)
$$

This is identical to the previous chapter giving the leading order dispersive solution as

$$
z_{0}=d+a \operatorname{cn}^{2}\left(k\left(X-\delta_{1}\right), m\right) .
$$

Where the slowly varying parameters are given as before

$$
a=-8\left(\omega_{1}^{\prime}\right)^{2} m^{2} k^{2}, \quad d=\frac{8}{3}\left(\omega_{1}^{\prime}\right)^{2} k^{2}\left(2 m^{2}-1\right), \quad k=\left(\frac{3(-c+\cos (\pi x))}{16\left(\omega_{1}^{\prime}\right)^{4}\left(m^{4}-m^{2}+1\right)}\right)^{\frac{1}{4}} .
$$

To resolve the parameters $\omega_{1}^{\prime}$ and $m$ rigorously, we once again impose periodicity,

$$
T=\frac{2 K(m)}{k}=\frac{4 K(m)\left(m^{4}-m^{2}+1\right)^{\frac{1}{4}}}{3^{\frac{1}{4}}\left(-c+\frac{1}{2} \cos (\pi x)\right)^{\frac{1}{4}}}
$$

and it is clear the expression for $\omega_{1}^{\prime}$ remains unchanged. While we commented on the importance of a constant period in the previous chapter, we will see that resolving the solvability condition rests on constant periodicity. The parameter $m$ at this point remains free and is used to remove secularity at the next order of the perturbative hierarchy given by

$$
\left(\omega_{1}^{\prime}\right)^{2} z_{1 X X}-\frac{3}{2} z_{0} z_{1}=-2 \omega_{1}^{\prime} \omega_{2}^{\prime} z_{0 X X}-2 \omega_{1}^{\prime} z_{0 X x}-\omega_{1}^{\prime \prime} z_{0 X}+\nu \int_{-1}^{1} \cos \pi(x+v) z_{0}(v, X) d v
$$

The integral term is now included in the analysis and presumably has some small effect on the modulation of the slowly varying parameters. For brevity we write this as

$$
L z_{1}=f(x, X)
$$

where $L$ is given by

$$
L=\left(\omega_{1}^{\prime}\right)^{2} \partial_{X}^{2}-\frac{3}{2} z_{0}
$$

Using the self-adjointness of $L$ and the fact that $z_{0 X}$ is still a homogeneous, periodic solution such that

$$
L z_{0 X}=\left(\omega_{1}^{\prime}\right)^{2} z_{0 X X X}-\frac{3}{2} z_{0} z_{0 X}=0,
$$

it follows from the Fredholm alternative that bounded periodic solutions $z_{1}$ to $L z_{1}=$ $f(x, X)$ exist if and only if

$$
\int_{0}^{T} f(x, X) z_{0 X} d X=0 .
$$


Substituting the expression for $f(x, X)$ yields

$$
\int_{0}^{T} 2 \omega_{1}^{\prime} \omega_{2}^{\prime} z_{0 X} z_{0 X X}+2 \omega_{1}^{\prime} z_{0 X} \psi_{0 X X}+\omega_{1}^{\prime \prime} z_{0 X}^{2}+\frac{\nu}{\sqrt{\lambda}}\left(\int_{0}^{2} \cos \pi(x+v) z_{0}(v, X) d v\right) z_{0 X} d X=0 .
$$

As before, noticing that

$$
\frac{d}{d x}\left(\omega_{1}^{\prime} z_{0 X}^{2}\right)=\omega_{1}^{\prime \prime} z_{0 X}^{2}+\omega_{1}^{\prime} \frac{\partial}{\partial X}\left(z_{0 X}^{2}\right)+2 \omega_{1}^{\prime} z_{0 X} z_{0 X x}
$$

implying by periodicity of $z_{0}$ that

$$
\frac{d}{d x} \omega_{1}^{\prime} \int_{0}^{T} z_{0 X}^{2} d X=\int_{0}^{T} \omega_{1}^{\prime \prime} z_{0 X}^{2}+2 \omega_{1}^{\prime} z_{0 X} z_{0 X x} d X
$$

the solvability condition can be reduced to

$$
\frac{d}{d x} \overbrace{\omega_{1}^{\prime} \int_{0}^{T} z_{0 X}^{2} d X}^{Q}+\frac{\nu}{\sqrt{\lambda}} \int_{0}^{T}(\underbrace{\int_{-1}^{1} \cos \pi(x+v) z_{0}(v, X) d v}_{I_{v}}) z_{0 X} d X=0 .
$$

The primary difference from this solvability condition from that found for the fKdV before is the Fredholm integral term. This itself is not surprising and, in fact, is by construction through the choice of $\nu=\mathcal{O}(\sqrt{\lambda})$. What is interesting, and more subtle, is that the presence of a Fredholm integral term appears to introduce a recursive problem. Although it was not carried out explicitly, in the previous analysis the first term in (4.11) could be computed explicitly using the expression for $z_{0}$ to obtain a nonlinear equation for $m$ in terms of $x$. The possibility of explicitly determining the first term remains unchanged, however, in order to compute $I_{v}$ explicitly (if it can be done at all) we need to know the dependence of the modulus $m$ on $x$ as each slowly varying parameter of $z_{0}$ depends on it. But the determination of the dependence $m$ on $x$ is the role of the solvability condition, of which $I_{v}$ is a part of and thus the problem. While it appears that $m$ finds itself in the same situation as the chicken and the egg, there is an avenue we can take to resolve this solvability condition presented in the following section. 


\subsection{Solvability}

As the first term in the solvability condition (4.11) can be determined analytically, we direct our concentration to resolving the second term

$$
\int_{0}^{T}(\underbrace{\int_{-1}^{1} \cos \pi(x+v) z_{0}(v, X) d v}_{I_{v}}) z_{0 X} d X
$$

into a function of $m$ and $x$. The principal idea here is that of separability. Supposing for a moment that $z_{0}(v, X)$ can be written in terms of functions $M_{i}(v)$ and $N_{i}(X)$ such that

$$
z_{0}=\sum_{i=0}^{\infty} M_{i}(v) N_{i}(X)
$$

then the integral $I_{v}$ can be written as

$$
I_{v}=\sum_{i=0}^{\infty} \int_{-1}^{1} \cos \pi(x+v) M_{i}(v) d v N_{i}(X) .
$$

This would, in principle, at least allow us to compute the $X$ integration in (4.12) in the form of an infinite series. This is not ideal in comparison to finding a closed form solution, but from a practical point of view, we will inherit the nonlinear equation from the first term of solvability regardless of the form of $I_{v}$, so computing the first $N$ terms of the infinite series should give an appropriate approximation of $m$. Before proceeding along these lines, we will need to borrow a lemma from Hereman et al. [20] extending the Cauchy Product Rule.

Lemma 4.3.1 Given a finite set of infinite series indexed by $i$ such that $F^{(i)}=\sum_{n=0}^{\infty} f_{n}^{(i)}$, then their finite product is given by

$$
\prod_{i=1}^{I} F^{(i)}=\sum_{n=0}^{\infty} \sum_{r=0}^{n} \ldots \sum_{m=0}^{k} \sum_{l=0}^{m} f_{l}^{(1)} f_{m-l}^{(2)} \ldots f_{n-r}^{(I)}
$$

Proof Beginning with $F^{(1)}=\sum_{n=0}^{\infty} f_{n}^{(1)}$ and $F^{(2)}=\sum_{n=0}^{\infty} f_{n}^{(2)}$ we find the Cauchy Product of the two to be

$$
F^{(1)} F^{(2)}=\sum_{n=0}^{\infty} \sum_{l=0}^{n} f_{l}^{(1)} f_{n-l}^{(2)} \triangleq \sum_{n=0}^{\infty} h_{n}^{(1)},
$$


where we have collected the sum $\sum_{l=0}^{n} f_{l}^{(1)} f_{n-l}^{(2)}$ into a single coefficient $h_{n}^{(1)}$. This allows us to reapply the Cauchy Rule to a third sum $F^{(3)}=\sum_{n=0}^{\infty} f_{n}^{(3)}$. Multiplying $F^{(1)} F^{(2)} F^{(3)}$ and using (4.14) gives

$$
F^{(1)} F^{(2)} F^{(3)}=\left(\sum_{n=0}^{\infty} h_{n}^{(1)}\right)\left(\sum_{n=0}^{\infty} f_{n}^{(3)}\right) \text {. }
$$

Using the Cauchy Product rule this becomes

$$
F^{(1)} F^{(2)} F^{(3)}=\sum_{n=0}^{\infty} \sum_{p=0}^{n} h_{p}^{(1)} f_{n-p}^{(3)}
$$

Substituting the definition of $h_{n}^{(1)}$, this can be re-written in terms of the original sequences to yield

$$
F^{(1)} F^{(2)} F^{(3)}=\sum_{n=0}^{\infty} \sum_{p=0}^{n} \sum_{l=0}^{p} f_{l}^{(1)} f_{p-l}^{(2)} f_{n-p}^{(3)} .
$$

Continuing this process for a finite product yields (4.13). xplus0emminus0.5em

This small lemma has been tremendously useful in terms of finding soliton solutions and kink-type solutions to nonlinear evolution equations prevalent in soliton theory [20, 11]. The question of the conditions and circumstance under which the new sum converges for general sequences $f^{(i)}$ falls outside the scope of this thesis. In the present case, we intend on multiplying together uniformly convergent Fourier series and as such, assume the product of these series leads to another convergent Fourier series although we will see that this question is not of great importance in the context of the present application.

We now look for a separable series representation of the cnoidal function. Fortunately, by virtue of being continuously differentiable in the real plane, the cnoidal function has a uniformly convergent Fourier series [35]. Defining the "nome" $q=e^{\frac{\pi K^{\prime}}{K}}$, where $K(m)$ is still the complete elliptic integral of the first kind and $K^{\prime}(m)=K(1-m)$, the Fourier series for the cnoidal function can be written (see [1]) as,

$$
\operatorname{cn}(x, m)=\frac{2 \pi}{m^{\frac{1}{2}} K} \sum_{n=0}^{\infty} \frac{q^{n+1 / 2}}{1+q^{2 n+1}} \cos \left\{(2 n+1) \frac{\pi x}{2 K}\right\} .
$$

Then using the Cauchy Product, the squared cnoidal function can be written as

$$
\mathrm{cn}^{2}(x, m)=\frac{4 \pi^{2}}{m K^{2}} \sum_{n=0}^{\infty} \sum_{l=0}^{n} A_{l, n-l}(q) \cos \left\{(2 l+1) \frac{\pi x}{2 K}\right\} \cos \left\{(2(n-l)+1) \frac{\pi x}{2 K}\right\}
$$


where,

$$
A_{l, n-l}(q)=\frac{q^{n+1}}{1+q^{2 l+1}+q^{2(n-l)+1}+q^{2 n+2}} .
$$

It follows directly that we can re-write our leading order solution $z_{0}$ as

$$
z_{0}=d(x)+\frac{4 \pi^{2} a(x)}{m K^{2}} \sum_{n=0}^{\infty} \sum_{l=0}^{n} A_{l, n-l}(x) \cos \left\{(2 l+1) \frac{\pi k(x)\left(X-\delta_{1}\right)}{2 K(m(x))}\right\} \cos \left\{(2(n-l)+1) \frac{\pi k(x)\left(X-\delta_{1}\right)}{2 K(m(x))}\right\}
$$

where $A_{l, n-l}$ depends on $x$ via $q$. Initially, it appears the argument of the cosine being function of both $x$ and $X$, resists separability. The key insight here is that we have imposed periodicity in (4.9) such that

$$
\frac{k(x)}{2 K(m(x))}=\frac{1}{T}
$$

and $\mathrm{T}$ is a constant. This allows us to write the above expression for $z_{0}$ in a separated double series as

$$
z_{0}=d(x)+\frac{4 \pi^{2} a(x)}{m K^{2}} \sum_{n=0}^{\infty} \sum_{l=0}^{n} A_{l, n-l}(x) \cos \{(2 l+1) \tilde{X}\} \cos \{(2(n-l)+1) \tilde{X}\},
$$

where $\tilde{X}=\frac{\pi\left(X-\delta_{1}\right)}{T}$. We're now in a position to pull the $X$ dependence out of the first, inner integral of (4.12). Substituting (4.18) into $I_{v}$ as delineated in (4.12) gives

$$
\begin{gathered}
I_{v}=\int_{-1}^{1} \cos \pi(x+v) z_{0}(v, X) d v=\int_{-1}^{1} \cos \pi(x+v) d(v) d v+ \\
\int_{-1}^{1} \cos \pi(x+v) a(v)\left(\frac{4 \pi^{2}}{m(v) K^{2}(m(v))} \sum_{n=0}^{\infty} \sum_{l=0}^{n} A_{l, n-l}(v) \cos \{(2 l+1) \tilde{X}\} \cos \{(2(n-l)+1) \tilde{X}\}\right) d v
\end{gathered}
$$

Expanding the function $\cos \pi(x+v)$ in the second integral and taking the summed cosine terms outside of the integral gives

$$
\begin{aligned}
& I_{v}=\int_{-1}^{1} d(v) \cos \pi(x+v) d v \\
& +\cos \pi x \sum_{n=0}^{\infty} \sum_{l=0}^{n} \tilde{A}_{l, n-l}^{(1)} \cos \{(2 l+1) \tilde{X}\} \cos \{(2(n-l)+1) \tilde{X}\} \\
& \quad-\sin \pi x \sum_{n=0}^{\infty} \sum_{l=0}^{n} \tilde{A}_{l, n-l}^{(2)} \cos \{(2 l+1) \tilde{X}\} \cos \{(2(n-l)+1) \tilde{X}\}
\end{aligned}
$$

where $\tilde{A}_{l, n-l}^{(1)}$ and $\tilde{A}_{l, n-l}^{(2)}$ are given by 


$$
\begin{aligned}
& \tilde{A}_{l, n-l}^{(1)}=4 \pi^{2} \int_{-1}^{1} \frac{\cos \pi v a(v) A_{l, n-l}(v)}{m(v) K^{2}(m(v))} d v \\
& \tilde{A}_{l, n-l}^{(2)}=4 \pi^{2} \int_{-1}^{1} \frac{\sin \pi v a(v) A_{l, n-l}(v)}{m(v) K^{2}(m(v))} d v
\end{aligned}
$$

and are constant in $x$ and $X$. With $I_{v}$ now separated, the first observation we make is that

$$
\int_{0}^{T} \int_{-1}^{1} \cos \pi(x+v) d(v) d v z_{0 X} d X=0
$$

by the periodicity of $z_{0}$ simplifying the expression (4.12) to

$$
\begin{aligned}
\int_{0}^{T}(\cos \pi x & \sum_{n=0}^{\infty} \sum_{l=0}^{n} \tilde{A}_{l, n-l}^{(1)} \cos \{(2 l+1) \tilde{X}\} \cos \{(2(n-l)+1) \tilde{X}\} \\
& \left.-\sin \pi x \sum_{n=0}^{\infty} \sum_{l=0}^{n} \tilde{A}_{l, n-l}^{(2)} \cos \{(2 l+1) \tilde{X}\} \cos \{(2(n-l)+1) \tilde{X}\}\right) z_{0 X} d X .
\end{aligned}
$$

We now represent $z_{0 X}$ by,

$$
\partial_{X} z_{0}=-\frac{8 \pi^{3} a(x)}{T m(x) K^{2}(m(x))}\left(\sum_{n=0}^{\infty} \frac{q^{n+1 / 2}}{1+q^{2 n+1}} \cos \{(2 n+1) \tilde{X}\}\right)\left(\sum_{n=0}^{\infty} \frac{(2 n+1) q^{n+1 / 2}}{1+q^{2 n+1}} \sin \{(2 n+1) \tilde{X}\}\right)
$$

Calling upon the result of Lemma 4.4.1 which, in the case of three sums gives

$$
\prod_{j=1}^{3} F^{(j)}=\sum_{n=0}^{\infty} \sum_{p=0}^{n} \sum_{l=0}^{p} f_{l}^{(1)} f_{p-l}^{(2)} f_{n-p}^{(3)}
$$

We take

$$
\begin{gathered}
f_{l}^{(1)}=\sum_{k=0}^{l} \tilde{A}_{k, l-k}^{(1)} \cos \{(2 k+1) \tilde{X}\} \cos \{(2(l-k)+1) \tilde{X}\} \\
f_{p-l}^{(2)}=\frac{q^{p-l+1 / 2}}{1+q^{2(p-l)+1}} \cos \{(2(p-l)+1) \tilde{X}\} \\
f_{n-p}^{(3)}=\frac{(2(n-p)+1) q^{n-p+1 / 2}}{1+q^{2(n-p)+1}} \sin \{(2(n-p)+1) \tilde{X}\} .
\end{gathered}
$$

It is clear we are now left with investigating the integrals of the form

$$
\int_{0}^{T} \cos \{(2 k+1) \tilde{X}\} \cos \{(2(l-k)+1) \tilde{X}\} \cos \{(2(p-l)+1) \tilde{X}\} \sin \{(2(n-p)+1) \tilde{X}\} d X
$$


The solution to the above integral is in fact always equal to zero making the expression (4.12) into the equality,

$$
\int_{0}^{T}(\underbrace{\int_{-1}^{1} \cos \pi(x+v) z_{0}(v, X) d v}_{I_{v}}) z_{0 X} d X=0 .
$$

Thus the final form of the solvability condition (4.11) is

$$
\omega_{1}^{\prime} \int_{0}^{T} z_{0 X}^{2} d X=C_{1}
$$

where $C_{1}$ is a constant as before. We can see immediately that the implication of the above solvability condition is that (4.1) with a weak bottom forcing has the same leading order solutions as the previous section with no bottom forcing.

From a physical standpoint, the orthogonality of $z_{0 X}$ and the Fredholm integral term suggests that the coupling effects of the bottom forcing and the wave speed are orthogonal to the wave speed within the boundary layer. This is not difficult to envision as the cnoidal/soliton waves arising from strong horizontal sloshing are particularly robust, self re-enforcing entities and their stability to small bottom forcing perturbations is not counterintuitive. Nevertheless, this chapter provides the mathematical verification of this and illustrates that even a small bottom forcing can present interesting analytical challenges.

\subsection{Comparison with Numerical Solutions}

Numerical simulations were run for the full forced KdV-Fredholm using a pseudo spectral method originally proposed by Fornberg and Whitham [15] described in Appendix A. Given the non-uniqueness of solutions to 4.1 subject to periodic boundary conditions and the mean condition, it is difficult to predict, for a given value of $\Delta$, which steady-state solution will be reached, for instance see Figure 3.3 in the previous chapter. In order to make comparisons with the analytical results we seed the pseudo-spectral solver with the steady-state result of interest as the initial value. What this does is coax the evolution to settle into the orbit(s) illustrated in Figure 3.2 from the previous chapter. Figure 4.1 illustrates the comparison of the double internal orbit asymptotic solution with numerical results and we find good agreement. One can see there are slight oscillations around the non-dispsersive solutions from the numerical calculations and we attribute this to 


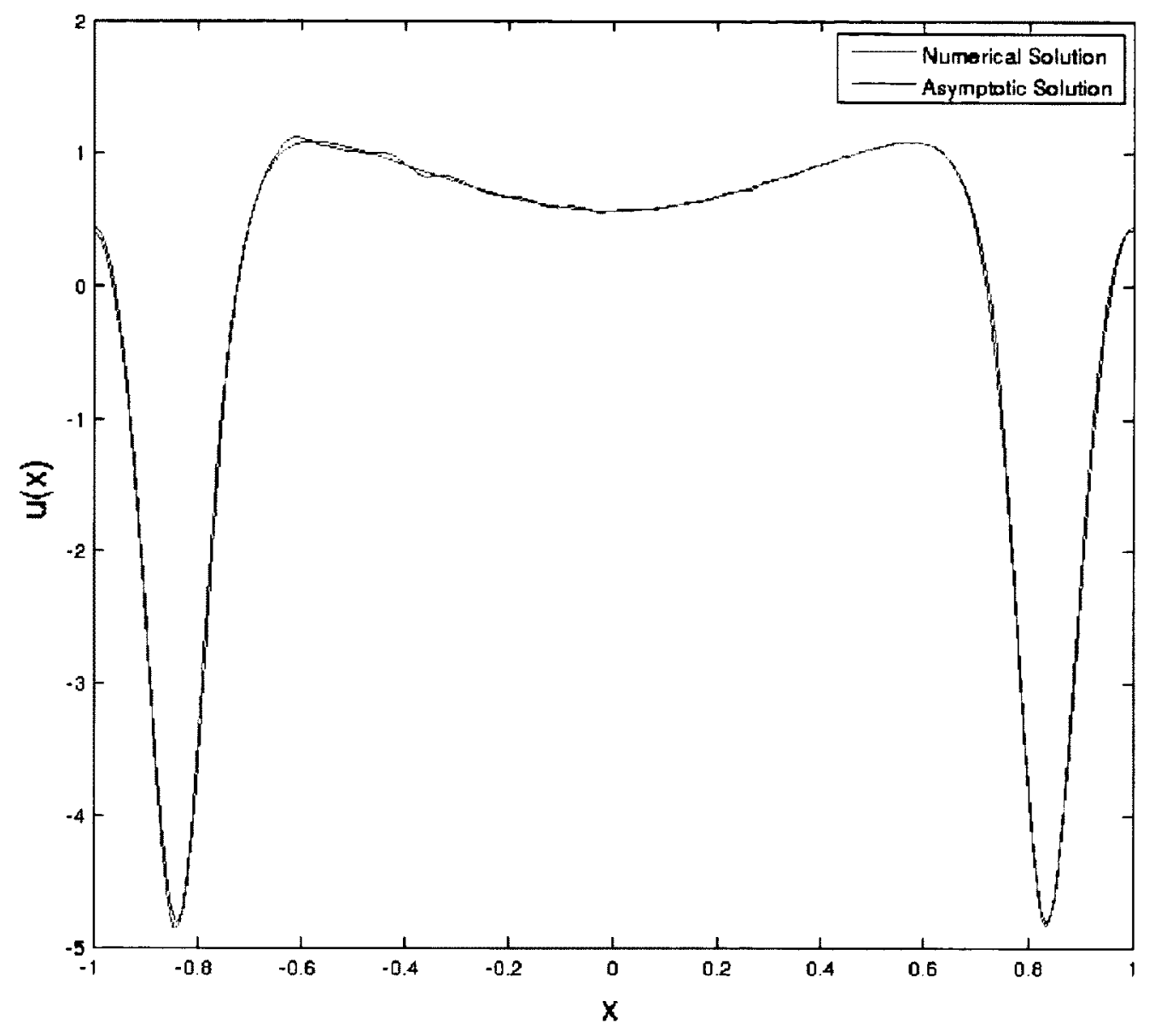

Figure 4.1: Comparison of a double internal orbit asymptotic solution with numerical results for $c=2$. The steep peaks are given by the inner solutions $z_{0}(x, X)$ which are matched to the slowly varying middle is $y_{+}$. A full description of how the asymptotic solution was plotted is contained in Chapter Three. The dependent variable $x$ corresponds to real time at the steady-state and solution is plotted over one period. The parameter values are $\lambda=0.005, \nu=0.0236$. The value of $\Delta$ is 0.8786 , which falls within the resonant band. 
proliferation of error around an unavoidable discontinuity from plotting the asymptotic solution described in Chapter Three. Recall that it is the asymptotic solution which is the initial value for the numerical simulation. The difficulty of representatively comparing asymptotic solutions with numerical solutions is perhaps the largest shortcoming of this thesis work and is discussed in the conclusion.

\subsection{Summary}

- Asymptotic solutions to the fKdV equation with a weak integral Fredholm term were found to be equivalent to the fKdV equation at leading order.

- The Fredholm-integral term was found to be orthogonal to the wave speed inside of the layer and consequently, the coupling effects orthogonal to the horizontal velocity. 


\section{Chapter 5}

\section{The Strong KdV-Fredholm}

\subsection{Introduction}

The previous chapter illustrated what kind of interesting mathematical quandaries arise with the inclusion of a weak integral term. To complete the study of nonlinear sloshing in an analytically tractable fashion we now turn towards the same equation as before in the absence of damping reproduced here,

$$
\lambda u^{\prime \prime}(x)=\frac{3}{4} u^{2}(x)+\nu \int_{-1}^{1} \cos \pi(x+v) u(v) d v+\cos (\pi x)-c
$$

under the assumption that $\lambda \ll 1$, subject to $u(x)=u(x-2)$ and the mean condition,

$$
\int_{-1}^{1} u(x) d x=\frac{4}{3} \Delta
$$

now with $\nu=\mathcal{O}(1)$. While standard techniques for differential equations are readily equipped to handle weak integral terms, introducing the Fredholm term with a new strength creates difficulties with existing multiple scales theory. This does, however, present an opportunity to develop some novel methodology.

In this chapter we study equation (5.1) with all terms save for $\lambda$ being order one. In section 5.2 we consider a general nonlinear Fredholm equation of the second kind and develop a method for solving it, which is then applied to find the non-dispersive solutions. In section 5.3, we outline a boundary layer technique for integro-differential equations and use it to find particular dispersive solutions. In section 5.4, we combine this boundary layer technique with the generalized multiple scales analysis used in the 
previous chapters to find multiple internal orbit solutions. Finally, in section 5.5 we compare analytic results with numerical ones.

\subsection{A Nonlinear Fredholm Equation}

The perturbative study of (5.1) with $\nu=\mathcal{O}(1)$ begins as usual by assuming a regular perturbative expansion,

$$
u(x)=\sum_{n=0}^{N} \lambda^{n} \tilde{y}_{0}(x),
$$

which yields the non-dispersive integral equation at leading order,

$$
\frac{3}{4} \tilde{y}_{0}^{2}+\nu \int_{-1}^{1} \cos \pi(x+v) \tilde{y}_{0} d v+\cos \pi x-c=0 .
$$

To re-cast this in a more familiar form, we let $y_{0}=\tilde{y}_{0}^{2}$ and only consider the positive square root giving,

$$
\frac{3}{4} y_{0}+\nu \int_{-1}^{1} \cos \pi(x+v) \sqrt{y_{0}} d v+\cos \pi x-c=0
$$

The equation above is an interesting case of a more general nonlinear fredholm equation,

$$
u(x)=f(x)-\gamma \int_{a}^{b} K(x, v) u^{p}(v) d v, \quad 0<p<1
$$

for which there seems to be little analytic work to go on. Authors have considered the case where $p \in \mathbb{Z}$, and have been able to find series solutions using semi-analytic methods such as the Homotopy Analysis Method or Adomians decomposition [17]. This particular equation, however, does not seem to have been considered and as such we seek to find some method to treat it analytically.

Aside from the purely mathematical opportunity (5.4) presents, there is an application in mind here as well. Recall from chapter 2 that we asymptotically studied the fKdV with a cosine forcing. One may notice that the analysis was able to accommodate more general periodic source terms aside from the simple trigonometric term considered. Application wise, this corresponds to being waves being forced horizontally by a general, 2-periodic function. In the present application, a more general 2-periodic function forcing the bottom would manifest itself in a more general kernel of the Fredholm integral term and 
thus studying (5.4) allows us to push forward the analytic theory of sloshing in a general manner.

There is an analysis incentive here as well. As mentioned previously, the goal of this thesis is to find an analytically tractable model of resonant sloshing with gravitational effects. Thus far, we have found the model and studied it using well-founded techniques and, as we will see, the full method developed in this chapter does not depart significantly from that analysis, that is, when searching for the dispersive solutions. Seeking the nondispersive solutions in the strongly forced bottom case is the single largest mathematical departure from the horizontally forced case. Thus treating the integral term in some generality serves not only to answer an open mathematical question, but directly applies to our application and allows us to develop a methodology in the most general possible sense.

In the following theorem we propose a analytic/numerical technique to handle (5.4) based upon finite separability. The technique finds its roots in linear, separable Fredholm equations of the second kind and in that respect it is not entirely novel [6]. Its application however, is novel, thus warranting the presentation of the method in the form of a theorem.

Theorem 5.2.1 Given (5.4) where $K(x, v)$ can be decomposed in terms of a set of basis functions $e_{i}(x) \in C([a, b], \mathbb{R})$ and continuous functions $N_{i}(v) \in C([a, b], \mathbb{R})$ such that,

$$
K(x, v)=\sum_{i=0}^{N_{k}} e_{i}(x) N_{i}(v)
$$

and $f(x)$ can be likewise decomposed into a finite set of basis functions such that,

$$
f(x)=\sum_{i=0}^{N_{f}} f_{i} \tilde{e}_{i}(x)
$$

Then a finite series solution of (5.4) can be found up to a system of nonlinear algebraic equations.

Proof Substituting the decomposition of $K(x, v)$ and $f(x)$ as given by, (5.5) and (5.6) respectively into (5.4) gives,

$$
u(x)=\sum_{i=0}^{N_{f}} f_{i} \tilde{e}_{i}(x)-\gamma \sum_{i=0}^{N_{k}} K_{i} e_{i}(x) \int_{a}^{b} N_{i}(v) u^{p}(v) d v
$$


Letting $\mathcal{S}_{i}=\left\{1,2, \ldots, N_{f}\right\}$ denote the entire collection of indices, we define the subset $\mathcal{S} \subset \mathcal{S}_{i}$ the collection of indices $i$ for which $e_{i}(x)=\tilde{e}_{i}(x)$. Without loss of generality, we assume $N_{f} \geq N_{k}$. The series solution is now sought using the ansatz,

$$
u(x)=\sum_{i=0}^{N_{f}} u_{i}^{(1)} e_{i}(x)+\left(1-\delta_{i S}\right) u_{i}^{(2)} \tilde{e}_{i}(x)
$$

where the notation $\delta_{i \mathcal{S}}$ denotes a modified Kronecker delta defined by

$$
\delta_{i S} \triangleq \begin{cases}1 & \text { if } i \in \mathcal{S} \\ 0 & \text { if } i \notin \mathcal{S}\end{cases}
$$

Substituting (5.8) into (5.7) gives

$$
\begin{aligned}
\sum_{i=0}^{N_{f}} u_{i}^{(1)} e_{i}(x)+ & \left(1-\delta_{i S}\right) u_{i}^{(2)} \tilde{e}_{i}(x)=\sum_{i=0}^{N_{f}} f_{i} \tilde{e}_{i}(x) \\
& -\gamma \sum_{i=0}^{N_{k}} e_{i}(x) \int_{a}^{b} N_{i}(v)\left(\sum_{k=0}^{N_{f}} u_{k}^{(1)} e_{k}(v)+\left(1-\delta_{i \mathcal{S}}\right) u_{k}^{(2)} \tilde{e}_{k}(v)\right)^{p} d v
\end{aligned}
$$

To determine the nonlinear system, we collect the coefficients of $e_{i}(x)$ and for $\tilde{e}_{i}(x)$ and equate to zero. One can see that for $u_{i}^{(2)}$ this implies that

$$
u_{i}^{(2)}=f_{i}, \quad \forall i \in \mathcal{S}_{i} .
$$

To determine each $u_{i}^{(1)}$ we first consider the set of indices, $\mathcal{U}_{1}=\left\{i: i \geq N_{k}\right\}$. Collecting coefficients of $e_{i}(x)$ and equating them to zero gives

$$
u_{i \in \mathcal{U}_{1}}^{(1)}=0 \text {. }
$$

Similarly for the set $\mathcal{U}_{2}=\left\{i: i \in \mathcal{S}, i \leq N_{k}\right\}$, using the definition of the modified kronecker delta and using $e_{i}(x)=\tilde{e}_{i}(x)$ for $i \in S$ we have

$$
u_{i \in \mathcal{U}_{2}}^{(1)}=f_{i}-\gamma \int_{a}^{b} N_{i}(v)\left(\sum_{k=0}^{N_{f}} u_{k}^{(1)} e_{k}(v)+\left(1-\delta_{i S}\right) u_{k}^{(2)} \tilde{e}_{k}(v)\right)^{p} d v .
$$

The final set of equations comes from the indices $\mathcal{U}_{3}=\left\{i: i \notin \mathcal{S}, i \leq N_{k}\right\}$, which after collecting coefficients as before gives the system

$$
u_{i \in \mathcal{U}_{3}}^{(1)}=\gamma \int_{a}^{b} N_{i}(v)\left(\sum_{k=0}^{N_{f}} u_{k}^{(1)} e_{k}(v)+\left(1-\delta_{i \mathcal{S}}\right) u_{k}^{(2)} \tilde{e}_{k}(v)\right)^{p} d v .
$$

As $\mathcal{U}_{1} \cup \mathcal{U}_{2} \cup \mathcal{U}_{3}=\mathcal{S}_{i}$, every coefficient of the ansatz is accounted for and this completes the proof. $\quad x$ plus0em minus0.5em 
The choice of naming this technique a analytical/numerical method lies in the weighty reliance on solving the nonlinear equations. Naturally, being able to do this by hand in general cases could present a formidable challenge and thus at some point, numerics will have to be involved. While this result is limited, it is readily equipped to handle appropriately truncated, delayed periodic kernels $K(t-s)$ and inhomogeneities $f(x)$ as required by our application. Furthermore, it appears to be the first analytical instance where the degree of nonlinearity within the integral is less than one has been treated. More immediately, the method is powerful when seeking the non-dispersive solutions to (5.2) presented in the next subsection.

\subsubsection{Non-dispersive Solutions}

Following Theorem 5.2.1, we look to solve (5.3) reproduced here,

$$
\frac{3}{4} y_{0}+\nu \int_{-1}^{1} \cos \pi(x+v) \sqrt{y_{0}} d v+\cos \pi x-c=0 .
$$

Expanding out the $\cos \pi(x+v)$ term gives,

$$
\frac{3}{4} y_{0}+\nu \cos \pi x \int_{-1}^{1} \cos \pi v \sqrt{y_{0}} d v-\nu \sin \pi x \int_{-1}^{1} \sin \pi v \sqrt{y_{0}} d v+\cos \pi x-c=0 .
$$

As the set of basis functions of the integral kernel and the inhomogeneity $\cos \pi x$ is $\{1, \cos \pi x, \sin \pi x\}$, we seek a solution with the ansatz

$$
y_{0}(x)=A+B \cos \pi x+C \sin \pi x .
$$

Immediately one can verify we must have $A=\frac{4}{3} c$. To determine $B$ and $C$ we equate the coefficients of $\cos \pi x$ and $\sin \pi x$ to zero giving the set of nonlinear equations,

$$
\begin{array}{r}
\frac{3}{4} B+\nu \int_{-1}^{1} \cos \pi v \sqrt{A+B \cos \pi v+C \sin \pi v} d v+1=0 \\
\frac{3}{4} C-\nu \int_{-1}^{1} \sin \pi v \sqrt{A+B \cos \pi v+C \sin \pi v} d v=0 .
\end{array}
$$

Initially there does not appear to be a closed form expression for the integrals; however, there is a quick simplification we can make. Examining the second equation closely, one can see that $C=0$ satisfies the second equation for any choice of $A$ and $B$ and thus the second equation can be eliminated from consideration. Noting that $c=\frac{4}{3} \tilde{A}$ and letting $B=-\frac{4}{3} B_{+}$the first equation simplifies to

$$
-\tilde{B}_{+}+\frac{2 \nu}{\sqrt{3}} \int_{-1}^{1} \cos \pi v \sqrt{\tilde{A}-B_{+} \cos \pi v} d v+1=0
$$


In this case there is a closed form expression for the integral giving the nonlinear equation dictating $B_{+}$in terms of $c$,

$$
\begin{aligned}
-B_{+}+\frac{8 \nu}{3 \sqrt{3} \pi B_{+}^{3}} & \sqrt{\frac{1}{c+B_{+}}}\left\{\frac{c^{2}}{B_{+}^{2}} K\left(\sqrt{2} \sqrt{\frac{B_{+}}{c+B_{+}}}\right)-K\left(\sqrt{2} \sqrt{\frac{B_{+}}{c+B_{+}}}\right)\right. \\
- & \left.\frac{c}{B_{+}} E\left(\sqrt{2} \sqrt{\frac{B_{+}}{c+B_{+}}}\right)-\frac{c^{2}}{B_{+}^{2}} E\left(\sqrt{2} \sqrt{\frac{B_{+}}{c+B_{+}}}\right)\right\}+1=0 .
\end{aligned}
$$

Where $K$ and $E$ are the complete elliptic integrals of the first and second kind respectively. We will find it convenient to define

$$
\begin{aligned}
K_{1}=\frac{8 \nu}{3 \sqrt{3} \pi B_{+}^{3}} \sqrt{\frac{1}{c+B_{+}}} & \left\{\frac{c^{2}}{B_{+}^{2}} K\left(\sqrt{2} \sqrt{\frac{B_{+}}{c+B_{+}}}\right)-K\left(\sqrt{2} \sqrt{\frac{B_{+}}{c+B_{+}}}\right)\right. \\
& \left.-\frac{c}{B_{+}} E\left(\sqrt{2} \sqrt{\frac{B_{+}}{c+B_{+}}}\right)-\frac{c^{2}}{B_{+}^{2}} E\left(\sqrt{2} \sqrt{\frac{B_{+}}{c+B_{+}}}\right)\right\},
\end{aligned}
$$

simplifying the nonlinear equation for $B_{+}$in relation to $c$ to

$$
-B_{+}+K_{1}+1=0 \text {. }
$$

This gives the first non-dispersive solution

$$
y_{+}=\frac{2}{\sqrt{3}} \sqrt{c-B_{+} \cos \pi x}
$$

One may think that the second solution to (5.2) is simply the negation of $y_{+}$but this is not the case. Using the form of $y_{+}$as an ansatz we substitute

$$
y_{-}=-\frac{2}{\sqrt{3}} \sqrt{c-B_{-} \cos \pi x}
$$

into (5.3) to get the nonlinear equation relating $B_{-}$to $c$,

$$
-B_{-}-K_{1}+1=0 \text {. }
$$

The question of whether or not there exist other solutions to (5.2) is a fairly intricate one. As far as Theorem 5.2.1 can reach, however, $y_{-}$and $y_{+}$is the limit to what we can determine. One can see these solutions bear an obvious kinsmen-ship with the previous non-dispersive solutions derived. However, the nonlinear integral equation does germinate new quantitative possibilities in the non-dispersive regime, namely non-uniqueness 


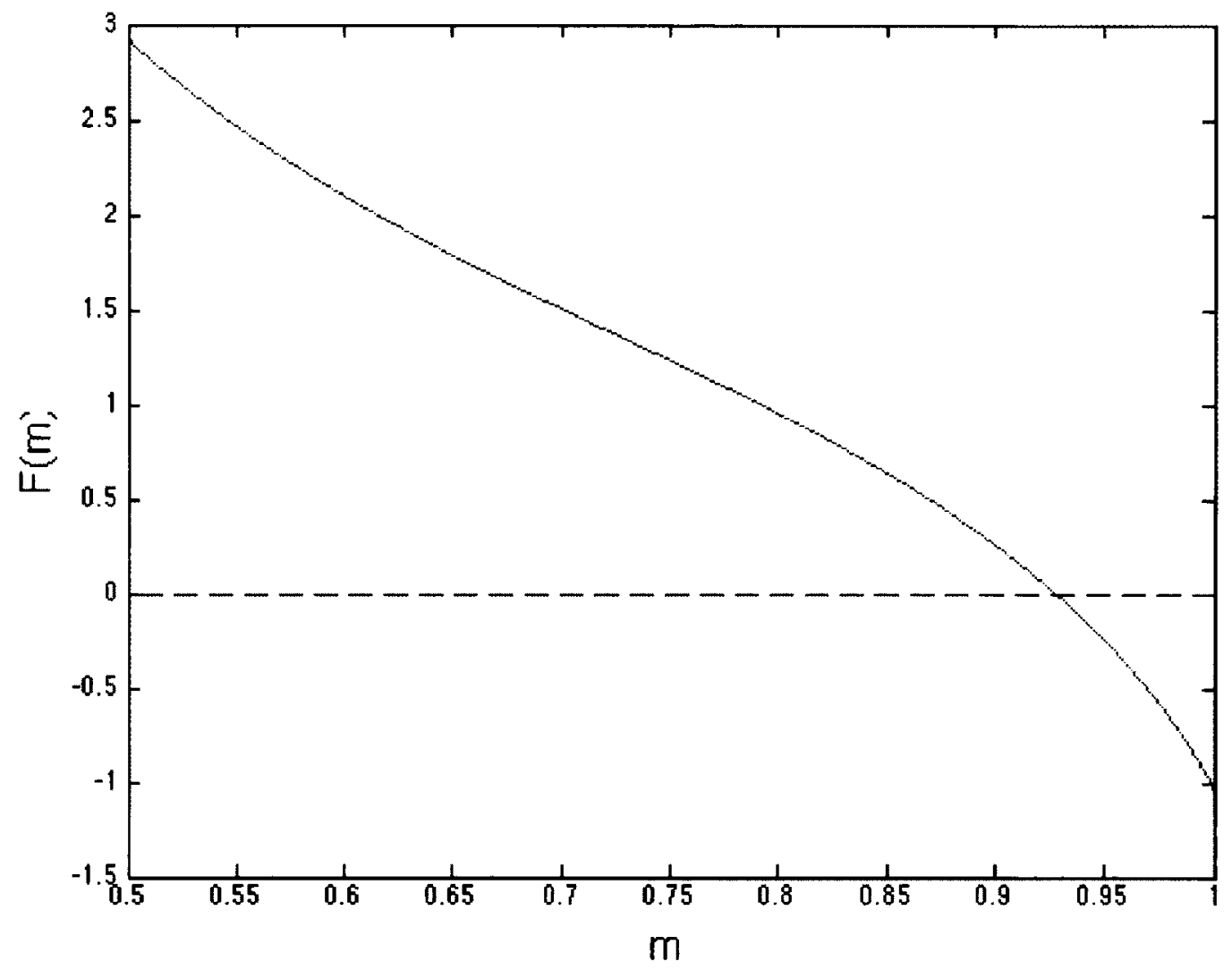

Figure 5.1: Plot of $F(m)$ dictating $B_{+}$. 


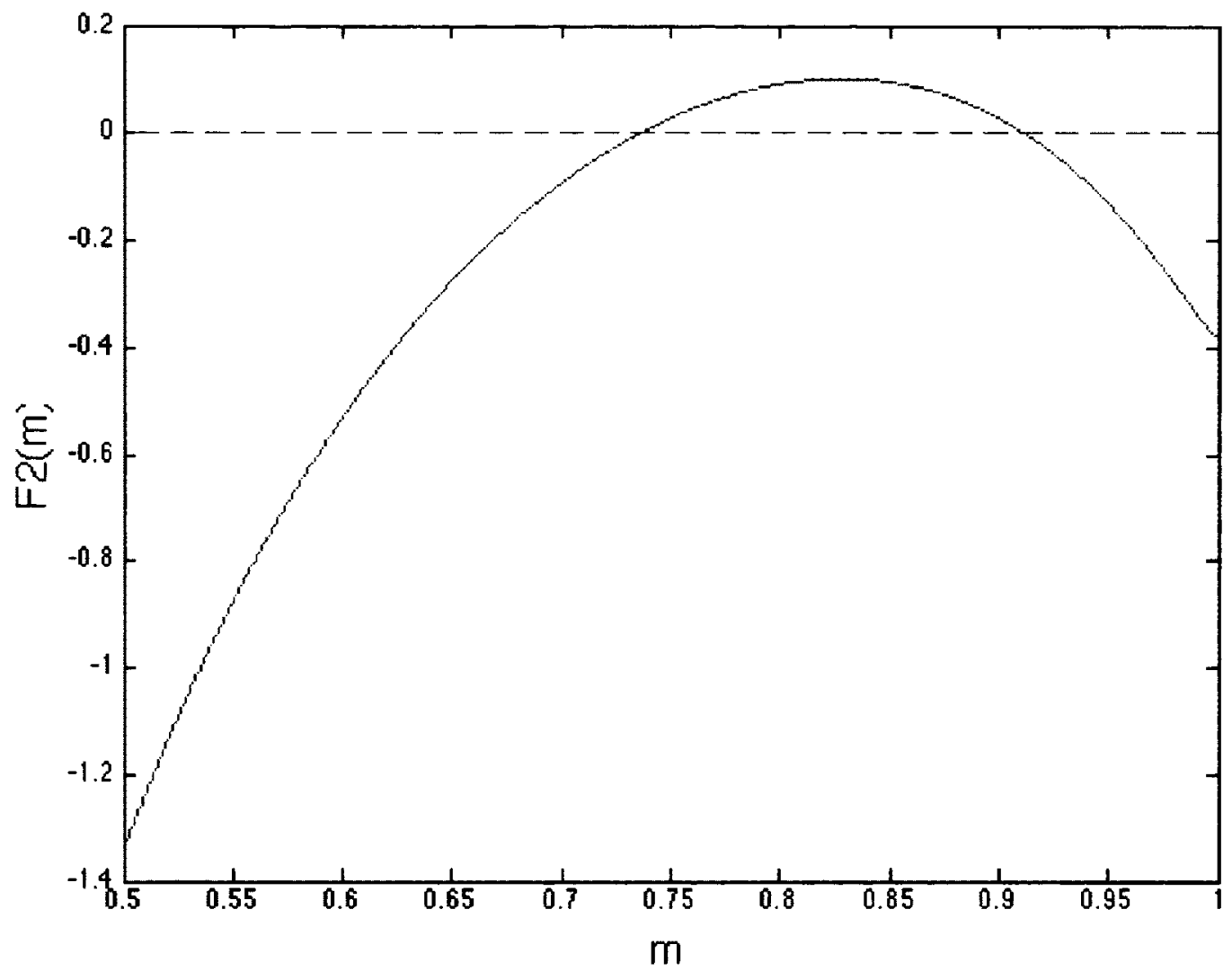

Figure 5.2: Plot of $F_{2}(m)$ dictating $B_{-}$. 
of the negative solutions. Figure 5.1 illustrates a representative plot of (5.17) for $c=2$ in terms of the elliptic modulus $m$ which is related to $B_{+}$by

$$
B_{+}=\frac{c m^{2}}{2-m^{2}} .
$$

and $F(m)=-B_{+}+K_{1}+1$. Only values of $m$ larger than 0.5 are shown as the graph diverges when $m \rightarrow 0$. The monotonicity of $F(m)$ suggests that there is a unique solution to (5.17) for a given $c$. In contrast, we can see that there are two solutions for $B_{-}$shown in Figure 5.2, where the relation between $B_{-}$and $m$ is the same as for $B_{+}$ and $m$ and $F_{2}(m)=-B_{-}-K_{1}+1$. We can furthermore find values of $c$ for which the maximum shown in Figure 5.2 dips below the zero line, and negative solutions cease to exist while positive solutions remain smooth. This is a significant departure from the purely horizontally forced case. Recall that previously, when the non-dispersive solutions ceased to exist, they did so for the same value of $c$. This made the resonant band symmetric, something which is now distorted by the presence of the integral term.

While it is interesting to explore the kind of resonant possibilities associated in the case in which two solutions for $y_{\text {- }}$ exist, we will leave this to future research and consider the case in which there exists a unique $y_{+}$and $y_{-}$. To move forward, we note that, although the resonant band may be asymmetric, we can establish its existence from constant sign nature argument in Chapter Three although clearly delineating the boundaries of the resonant band poses significant algebraic difficulty due to (5.17). Regardless, the constant sign nature argument of Chapter Three implies there will be a limited range of $\Delta$ which the non-dispersive solutions can satisfy and thus rapidly varying solutions must be sought to find solutions to (5.1) corresponding to $\Delta$ in a neighbourhood of zero. This is accomplished in the following sections.

\subsection{Boundary Layer Analysis}

\subsubsection{Premlinaries}

Having established the existence of a resonant band, we anticipate that within rapidly varying solutions reside. The treatment of the full integro-differential equation (5.1) will require an alternative but related form of analysis used in the preceding sections. To develop this method, we first seek boundary layer solutions for the single orbit cases in which the solution leaves the non-dispersive solution $y_{+}$and returns after a single loop, illustrated on the left of Figure 3.2. The location of the layer requires a subtle symmetry 
argument of Amundsen et al. [3]. One can see from the phase plane that, once the single orbit solution departs, in order to match with $y_{+}$again, $y_{+}$must be in the same place it was before. As the layer is of $\mathcal{O}(\sqrt{\lambda})$ thickness around its location $\delta, y_{+}$will only have identical values at $\delta \pm \sqrt{\lambda}$ if $\delta$ is located at a maxima or minima of $y_{+}$. Thus single internal orbits will arise at $x= \pm 1$ and external orbits will arise at $x=0$.

To find these single orbit solutions we will need to employ the boundary layer analysis of integro-differential equations. To illustrate the need for an alternative boundary layer analysis in the context of integral equations, we show the matching issues of classical boundary layer analysis in the context of equation (5.1). To find the leading order outer solution to (5.1), we expand $u(x)$ into a regular perturbation series

$$
u(x)=\sum_{n=0} \lambda^{n} y_{n}(x),
$$

forcing $y_{0}(x)$ to satisfy

$$
\frac{3}{4} y_{0}^{2}+\nu \int_{-1}^{1} \cos \pi(x+v) y_{0} d v+\cos \pi x-c=0,
$$

for which we found the solutions to be $y_{ \pm}$. Proceeding to seek a boundary layer solution using classical theory by letting $u(x)=U(X)$ where $X=\frac{x-\delta}{\sqrt{\lambda}}, \delta$ the location of the boundary layer, gives a leading order balance of

$$
U^{\prime \prime}(x)=\frac{3}{4} U^{2}+\nu \int_{-1}^{1} \cos \pi(x+v) d v U(X)+\cos \pi \delta-c .
$$

The integral term is readily verified as zero and the solution can be written as the wellknown soliton,

$$
U(X)=\frac{2}{\sqrt{3}} \sqrt{\cos \pi \delta-c}\left(1-3 \operatorname{sech}^{2}\left[\frac{(3 \cos \pi \delta-c))^{\frac{1}{4}}}{2} X\right]\right) .
$$

The issue now arises from matching. Taking the limit of the outer solution as it enters the boundary layer gives $\lim _{x \rightarrow \delta} y_{+}(x)=y_{+}(\delta)$, but taking the limit of the inner solution as it exits the boundary layer gives

$$
\lim _{X \rightarrow \pm \infty} U(X)=\cos \pi \delta-c
$$

Thus, unless by some coincidence $y_{+}(\delta)=\cos \pi \delta-c$, the boundary layer method of matched asymptotics is inadequate to determine the single orbit solutions. The question 
is why does this occur? Heuristically speaking, the success of the method of matched asymptotic expansions is rooted in derivatives exhibiting local behaviour whereas integrals are by nature, global. Thus, examining local behaviour in the context of an integral equation is inappropriate as the integral term relies on information collected along the entire domain. How then does one determine rapidly varying solutions from integral equations perturbatively? A method developed by Angell and Olmstead $[4,5]$ outlines a straightforward, boundary layer method for solving singularly perturbed problems of Volterra. While these equations differ slightly from ours, the technique is readily extendable. The remedy, it seems, is to seek a uniformly valid boundary layer solution a priori rather than a posteriori. We introduce this method in the following subsection.

\subsubsection{Singularly Perturbed Volterra Equations}

The simplest setting to introduce the method of Angell and Olmstead $[4,5]$ is in integral equations. We take time to note, however, that these ideas extend naturally to integrodifferential equations [5]. Consider the singularly perturbed, nonlinear Volterra equation of the second kind,

$$
\varepsilon u(x)=\int_{0}^{x} \frac{k(x-s)}{(x-s)^{\beta}} F[u(s), s] d s, \quad 0<\beta<1, \quad t \geq 0,
$$

where the delayed function $k(x-s)$ is analytic and the nonlinearity $F[u(s), s]$ is sufficiently well behaved to ensure well-posedness. Here the boundary layer is assumed to be at $x=0$ and so by introducing the inner variable

$$
X=\frac{x}{\varepsilon^{\alpha}},
$$

it follows that we can re-write the integral equation (5.22) in terms of $X$ to get,

$$
\varepsilon u(x)=\varepsilon^{\alpha(1-\beta)} \int_{0}^{x} \frac{k(0)+\varepsilon^{\alpha} k^{\prime}(0)(X-\sigma)+o\left(\varepsilon^{2 \alpha}\right)}{(X-\sigma)^{\beta}} F\left[u, \varepsilon^{\alpha} \sigma\right] d \sigma,
$$

where $\sigma=\varepsilon^{-\alpha} s$. In order to balance the external term $u(x)$ with the integral term $\alpha$ must be taken such that

$$
\alpha=(1-\beta)^{-1} .
$$

Having the relevant asymptotics in places, the approximate solution is then assumed to be a finite series of linear combinations of outer solutions $y_{i}(x)$ and $z_{i}(X)$ in $\varepsilon$ such that

$$
u(x)=\sum_{i=0}^{N} \varepsilon^{i}\left(y_{i}(x)+z_{i}(X)\right) .
$$


This immediately imposes the criterion

$$
\lim _{X \rightarrow \infty} z_{i}(X)=0,
$$

in order to ensure a match. One can see from the choice of ansatz that the goal is to seek uniformly valid solutions. In order to seek an outer solution, we substitute (5.23) into (5.22) and write the equation in terms of the slow variable $x$, giving to leading order,

$$
\varepsilon\left(y_{0}(x)+z_{0}\left(\frac{x}{\varepsilon^{\alpha}}\right)\right)=\int_{0}^{x} \frac{k(x-s)}{(x-s)^{\beta}} F\left[y_{0}(s)+z_{0}\left(\frac{s}{\varepsilon^{\alpha}}\right), s\right] d s .
$$

Fixing $x$ and taking the limit as $\varepsilon \rightarrow 0$ as well as using the imposition (5.24) gives

$$
\int_{0}^{x} \frac{k(x-s)}{(x-s)^{\beta}} F\left[y_{0}(s), s\right] d s=0 .
$$

This forces the leading order outer solution $y_{0}(x)$ to solve the nonlinear equation

$$
F\left(y_{0}(x), x\right)=0 .
$$

To move forward to the analysis of finding boundary layer solutions, we assume there exists one solution to this equation $y_{0}(x)=y(x)$ with properties consistent with the physics of the problem. Angell and Olmstead [4] found this assumption to be valid over a wide range of problems a posteriori. To seek an inner solution, (5.25) is re-written in the fast variable using the choice of $\alpha$ and expanding $k(t-s)$ to get at leading order

$$
y_{0}\left(\varepsilon^{\alpha} X\right)+z_{0}(X)=\int_{0}^{X} \frac{k\left(\varepsilon^{\alpha}(X-\sigma)\right)}{(X-\sigma)^{\beta}} F\left[y_{0}\left(\varepsilon^{\alpha} X\right)+z_{0}(\sigma), \varepsilon^{\alpha} \sigma\right] d s .
$$

Similarly taking the limit as $\varepsilon \rightarrow 0$ and fixing $X$ as before gives

$$
y_{0}(0)+z_{0}(X)=\int_{0}^{X} \frac{k(0)}{(X-\sigma)^{\beta}} F\left[y_{0}(0)+z_{0}(\sigma), 0\right] d \sigma
$$

the solution to which can be found using the wide literature on Volterra equations [4]. The single, principle idea here is the re-writing of the equation in terms of the fast variable. This properly addresses the interesting ambiguity of introducing a fast variable. In standard boundary layer analysis, $X$ and $x$ are treated as independent due to the local nature of derivatives. In reality they are still related closely enough that functions of each variable should be integrated. Thus rather than the fast solution $z(X)$ being pulled out of the integral as it was previously, information is collected with respect to the fast variable over the entire boundary layer. This is clearly consistent with singular perturbation 
theory for differential equations and generalizes it naturally to integral equations. This simple and elegant technique was applied to a multitude of integro-differential equations with a high order of accuracy and is well suited to find the external and internal orbits of the integro-differential equation (5.1), which we present in the next subsection.

\subsubsection{Single Orbit Solutions}

Knowing the location of the boundary layer and the non-dispersive solutions, determining the dispersive solutions becomes a straightforward application of Angell and Olmstead's technique just described. As the analysis for the single orbit solution and external orbit solution is much the same, we derive solutions for a general boundary layer location $\delta$ and substitute the locations for the external and internal orbits afterwards. Concerning ourselves with the leading order solution only, we substitute the ansatz

$$
u(x)=y_{+}(x)+z(X)
$$

where $X=\frac{x-\delta}{\sqrt{\lambda}}, y_{+}$is given by (5.18) and $z(X)$ decays to zero as $X \rightarrow \pm \infty$, into (5.1) giving

$$
\begin{aligned}
\lambda y_{+}^{\prime \prime}(x)+z^{\prime \prime}(X)=\frac{3}{4} y_{+}^{2}(x)+\frac{3}{2} y_{+} & (x) z(X)+\frac{3}{4} z^{2}(X) \\
& +\nu \int_{-1}^{1} \cos \pi(x+v)(y(x)+z(\sigma)) d v+\frac{1}{2} \cos \pi x-c .
\end{aligned}
$$

By virtue of $y_{+}$being an outer solution, the equation it satisfies can be pulled out and the above can be rewritten in terms of the fast variable as

$$
\begin{aligned}
\lambda y_{+}^{\prime \prime}(\sqrt{\lambda} X+\delta)+z^{\prime \prime}(X)=\frac{3}{2} y_{+}(\sqrt{\lambda} X & +\delta) z(X)+\frac{3}{4} z^{2}(X) \\
& +\sqrt{\lambda} \nu \int_{-\frac{1}{\sqrt{\lambda}}+\delta}^{\frac{1}{\sqrt{\lambda}}+\delta} \cos \pi(\sqrt{\lambda} X+\sqrt{\lambda} \sigma+2 \delta) z(\sigma) d \sigma .
\end{aligned}
$$

Here we can see that integral term has been shifted to the next order of $\lambda$. To take the limit as $\lambda$ goes to zero comfortably we need to enforce that the integral term remain order one, so in taking the limit as $\lambda \rightarrow 0$ this implies

$$
\lim _{\lambda \rightarrow 0} \nu \int_{-\frac{1}{\sqrt{\lambda}}+\delta}^{\frac{1}{\sqrt{\lambda}}+\delta} \cos \pi(\sqrt{\lambda} X+\sqrt{\lambda} \sigma+2 \delta) z(\sigma) d \sigma=\lim _{\lambda \rightarrow 0} I_{z}=\mathcal{O}(1) .
$$

There are two aspects of this analysis we are in a position to comment on. First, for our particular equation with the ansatz (5.29) as well as the impositions (5.24), (5.30) placed 
on $z(X)$, the singular perturbation analysis is able to separate the integro-differential equation into an outer integral equation and inner differential equation. Furthermore, we can see a departure from classic boundary layer analysis in that we are forced to impose a type of nonlinear mean condition in order to ensure well orderedness of the asymptotic series. Continuing, we fix $X$ and take the limit $\lambda \rightarrow 0$ giving the leading order inner solution equation,

$$
z^{\prime \prime}(X)=\frac{3}{2} y_{+}(\delta) z(X)+\frac{3}{4} z^{2}(X)
$$

which can be solved to get the soliton,

$$
z(X)=-\frac{3}{2} y_{+}(\delta) \operatorname{sech}^{2}\left(\frac{\sqrt{3 y_{+}(\delta)}}{4} X\right)
$$

This soliton solution has the property (5.24). To show that (5.30) is satisfied is straightforward. By the evenness of $z(X)$ we can see that $I_{z}$ defined in (5.30) is bounded above by the integral

$$
I_{z} \leq 3 y_{+}(\delta) \nu \cos \pi(\sqrt{\lambda} X+2 \delta) \int_{0}^{\frac{1}{\sqrt{\lambda}}+\delta} \operatorname{sech}^{2}\left(\frac{\sqrt{3 y_{+}(\delta)}}{4} \sigma\right) d \sigma .
$$

Evaluating this explicitly and taking the limit $\lambda \rightarrow 0$ gives the bound,

$$
\lim _{\lambda \rightarrow 0} I_{z} \leq 4 \sqrt{3 y_{+}(\delta)} \nu \cos (2 \pi \delta)
$$

Justifying the assumption that the integral remains order one. Heuristically speaking, this should always be the case unless the boundary layer solution is unbounded. So we note that these soliton solutions break down for an unbounded $c$. Accepting this limitation, we have our first leading order, single orbit resonant solutions given by,

$$
u(x)=y_{+}-\frac{3}{2} y_{+}(\delta) \operatorname{sech}^{2}\left(\frac{\sqrt{3 y_{+}(\delta)}}{4} X\right)
$$

where $\delta= \pm 1$ for internal orbits and $\delta=0$ for the external orbit. One can notice that overall the methodology departs only slightly from that of Amundsen et al.[3]. However, the subtle argument of pursuing a uniformly valid boundary layer solution a priori is not an obvious one and finds itself tremendously useful in this context. From the previous analysis, we can conjecture without going into detail that the single soliton solutions will only be able to satisfy limited values of $\Delta$ in a neighbourhood of zero. In general we will need multiple orbit solutions in order to explore behaviour across the entire resonant 
band. In order to do this, we combine Amundsen et al.'s [3] multiple-scale argument with the integral arguments of Angell and Olmstead [4] into an approach capable of finding multi-peak solutions. To the author's knowledge, this is a novel technique, presumably as this is the first instance it is necessary.

\subsection{Multiple orbit Solutions}

The general methodology to constructing asymptotic, multiple orbit solutions for singularly perturbed integro-differential equations in the form of equation (5.1) is, to the authors knowledge novel. This can be viewed as a natural generalization of the LukeKuzmak method $[25,24]$ for ordinary differential equations to integro-differential equations. The core idea of the method is to combine the multiple scales technique of Amundsen et al. [3] and the integral equation boundary layer analysis of Angell and Olmstead $[4,5]$. The method is simple and flexible and is outlined as follows.

1. Derive leading order outer solutions by introducing a regular perturbation expansion and solving the leading order equation.

2. Derive leading order inner solutions in two steps. Introduce a uniformly valid ansatz as in Angell and Olmstead [4], where the rapidly varying solution contains the generalized multiple scales argument of Luke-Kuzmak [25, 24] and decays to zero outside of the layer as in Angell and Olmstead [4]. Re-write any integral terms in terms of the fast variables while leaving the slowly varying scale untouched.

3. Remove secularities to determine remaining, slowly varying parameters by means of the Fredholm Alternative as in Luke [25] as well as Amundsen et al. [3]

The method is best made clear by applying it to equation (5.1) which appears to be the first equation for which such a methodology is necessary and which is presently not amenable to other forms of asymptotic analysis. Having already found the non-dispersive outer solutions as prescribed in step one of the method, we pursue the inner solution by introducing the generalized multiple scales argument as before defining

$$
X=\frac{\omega_{1}(x)}{\sqrt{\lambda}}+\omega_{2}(x)+\ldots
$$

The expansion for $u(x)$ is now taken as the uniformly valid ansatz described in step two given by

$$
u(x)=y_{+}+z_{0}(x, X)+\sqrt{\lambda}\left(y_{1}(x)+z_{1}(x, X)\right),
$$


subject to the rapid solution decay criterion,

$$
\lim _{X \rightarrow \pm \infty} z_{i}(x, X)=0
$$

The relevantly truncated operator transformation remains the same and is given by

$$
\frac{d^{2}}{d x^{2}} \rightarrow \frac{\left(\omega_{1}^{\prime}\right)^{2}}{\lambda} \partial_{X}^{2}+\frac{1}{\sqrt{\lambda}}\left(2 \omega_{1}^{\prime} \omega_{2}^{\prime} \partial_{X}^{2}+2 \omega_{1}^{\prime} \partial_{x} \partial_{X}+\omega_{1}^{\prime \prime} \partial_{X}\right)+\ldots
$$

As we are looking for solutions within the boundary layer, we re-write the integral in terms of the fast variable but leave the slowly varying functions in terms of the slowly varying variable. As mentioned, this is the natural extension of the multiple-scale analysis in the context of integro-differential equations. Re-writing the integral serves to collect information over the entire domain while leaving the slow variable unchanged serves to separate scales. This gives the leading order dispersive problem,

$$
z_{0 X X}(X)=\frac{3}{2} y_{+}(x) z_{0}(x, X)+\frac{3}{4} z_{0}^{2}(x, X)
$$

where the integral term appears at the next order in the hierarchy. The leading order dispersive problem admits the cnoidal solution,

$$
z_{0}(x, X)=d(x)+a(x) \mathrm{cn}^{2}\left(k(x)\left(X-\delta_{1}\right)\right)
$$

Where the slowly varying parameters are slightly different from before, given by

$$
a=-8\left(\omega_{1}^{\prime}\right)^{2} m^{2} k^{2}, \quad d=\frac{8}{3}\left(\omega_{1}^{\prime}\right)^{2} k^{2}\left(2 m^{2}-1\right)-y_{+}(x), \quad k=\left(\frac{3\left(c-B_{+} \cos (\pi x)\right)}{16\left(\omega_{1}^{\prime}\right)^{4}\left(m^{4}-m^{2}+1\right)}\right)^{\frac{1}{4}}
$$

We are still left with the free parameters $\omega_{1}^{\prime}$ and $m(x)$ which we find as before by removing secularities.

\subsubsection{Removing Secularity and Physical Discussion}

To remove secularities, we apply periodicity and the Fredholm alternative as in the previous chapters. The need for a constant period within the layer remains the same and thus we apply

$$
T=\frac{2 K(m)}{k(x)}
$$


where $T$ is chosen as $T=\frac{3^{\frac{1}{4}}}{4}$ as previously. To remove secularity at the next order, we once again apply the Fredholm alternative as in the preceding chapters (for the full process see, for instance, Section 4.3). Omitting the details, this produces

$$
\frac{d}{d x}\left(\omega_{1}^{\prime}(x) \int_{0}^{T} z_{0 X}^{2} d X\right)+\nu \int_{0}^{T} \int_{-\frac{1}{\sqrt{\lambda}}+\delta}^{\frac{1}{\sqrt{\lambda}}+\delta} \cos \pi(x+\sqrt{\lambda} \sigma) z(x, \sigma) d \sigma z_{0 X}(x, X) d X=0 .
$$

What we can see immediately is that the second term is zero by virtue of the inner integral producing a function of solely the slow variable $x$, making the final solvability condition identical to the previous ones

$$
\omega_{1}^{\prime}(x) \int_{0}^{T} z_{0 X}^{2} d X=C_{3}
$$

albeit with a different solvability constant. Here we pause to comment on the analysis in Chapter Four used to handle the solvability condition. Recall the integral term also appeared at the next order of the perturbative hierarchy and was likewise zero although it was substantially more involved to show. Interestingly, a strong integral term shares the same characteristic. One can see that using the same matching arguments used in the previous sections, ie. by imposing $m \rightarrow 1$ as $X \rightarrow \infty$, then $\lim _{X \rightarrow \pm \infty} z_{0}(x, X)=0$ satisfying (5.34). We are then finished, having the relevant modulation equations and impositions on the straggling constants as before. Note in the case that $\nu$ is either weak or zero, this collapses into the analysis of the two previous chapters.

Overall, the departure from Amundsen et al. in terms of finding dispersive solutions is small. In fact, in the analysis of Chapter 2 we could have used the same uniformly valid ansatz to recover the same solution found for the $\mathrm{fKdV}$ equation. The main difference is that this ansatz is required to handle the integral term and is not just an equivalent alternative. In other words, the seemingly innocuous choice of either pursuing a uniformly valid solution initially or a posteriori has profound implications for integral equations.

To compare with the fKdV equation, the asymptotic solutions of the forced $\mathrm{KdV}$ Fredholm equation are effectively the same with the exception of the non-dispersive solutions. What this illustrates mathematical is that forcing the bottom resonantly effectively acts as a secondary horizontal forcing. Physically, this illustrates the major fluid mechanical finding of this work. That theconsequence of the resonantly forcing the bottom is effectively the same as adding an additional horizontal forcing or that the qualitative behaviour of the motion of an inviscid fluid in a tank that is vertically 
and horizontally forced is the same as a horizontally forced tank. Having obtained the multiple orbit solutions, we culminate the study with comparison to numerical results.

\subsection{Comparison with Numerical Results}

As described in Chapter 4, the analytic results were compared to the full evolution by seeding the evolutionary code with the particular orbit as its initial condition. Figure 5.3 shows the comparison of the single internal orbit soliton approximation and the steadystate numerical results and we find excellent agreement between the two. For the multiple orbit comparison we choose two peaks to compare with numerical results, presented in Figure 5.4 and again we observe strong agreement. The small oscillations arising around the non-dispersive solution were described in Chapter 4 and are attributed to proliferation of error around an unavoidable discontinuity of the asymptotic solution. 


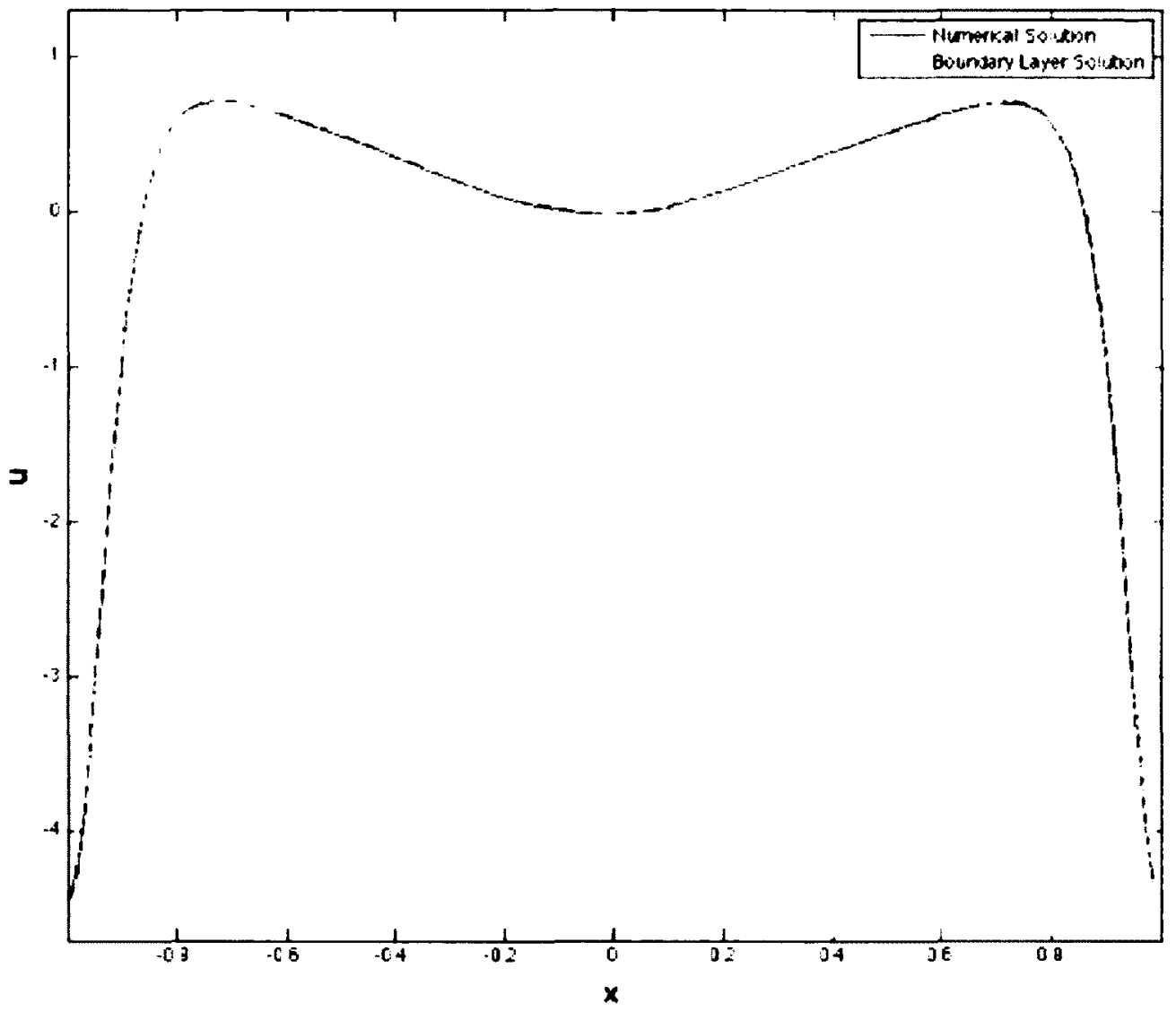

Figure 5.3: Comparison of an undamped, single orbit soliton approximation $u=y_{+}+$ $z_{0}(x, X)$ with numerical solution for $c=1.5$. Parameter values are $\lambda=0.005, \nu=1$ and $\Delta=1.8972$. A full description on how to plot $u(x)$ is given at the end of Chapter Three. 


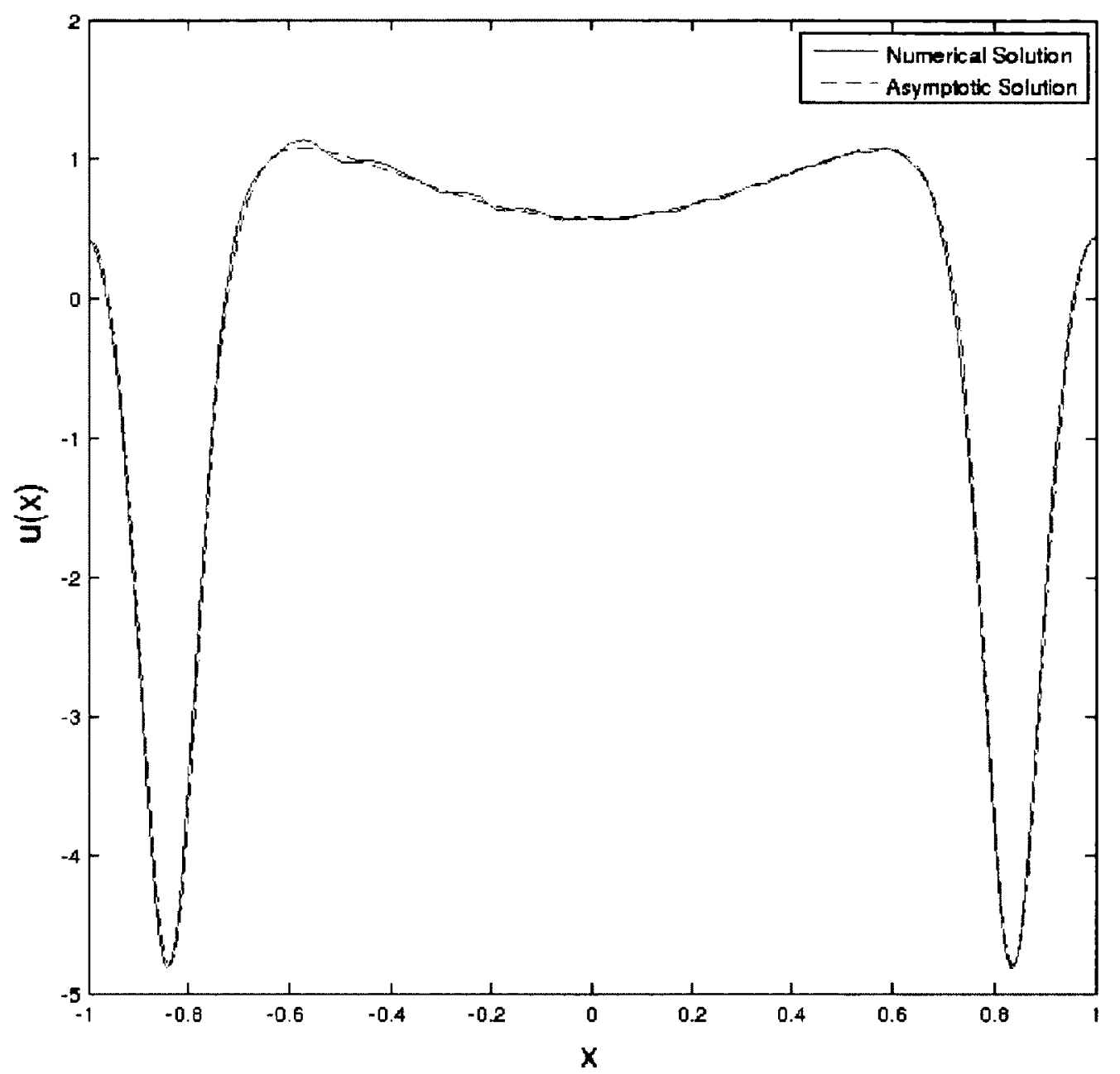

Figure 5.4: Comparison of an undamped, two orbit approximation $u(x)=y_{+}+z_{0}(x, X)$ with numerical solution for $c=2$. Parameter values are $\lambda=0.005, \nu=1$ and $\Delta=0.8370$. Note that the value of $\Delta$ for the double peak is smaller than the for the single peak plotted in Figure 5.3 and thus deeper within the resonant band. 


\section{Chapter 6}

\section{Conclusion}

\subsection{Summary of Results}

1. The forced KdV-Fredholm equation was shown to govern the location of the free surface for a fluid in a container experiencing resonant horizontal and vertical forcing. This was possible by using the transitivity of Cox and Mortells [12] singular perturbation argument, which bypasses the ill-posedness problem of Laplace's equation and the leading order boundary conditions.

2. The steady-state forced KdV-Fredholm equation was then analyzed in three cases: the case of no Fredholm term or simply the fKdV equation, the case of a weak Fredholm term and the case of a strong Fredholm term. The fKdV equation was previously studied by Amundsen et al. and their work was reviewed. The case of the weak Fredholm term was shown to have the same leading order solutions as the fKdV equation. The strong KdV Fredholm was shown to have similar qualitative behaviour to the $\mathrm{fKdV}$ but required the development and inclusion of additional analytic tools. The asymptotic analysis agreed well with numerical results.

\subsection{Summary of Contributions}

1. We discovered a novel equation type in the KdV-Fredholm.

2. We showed that a particular class of nonlinear Fredholm equations of the second kind can be reduced to a system of nonlinear equations. The method rests on the separability of the kernel and that the inhomogeneity can be decomposed into a 
set of basis functions. In general, this methodology can be likened to the solution technique for linear inhomogeneous Fredholm equations of the second kind and is not considered entirely novel. It does, however, treat a class of equations that to date, do not appear to have been considered.

3. A methodology development was made by combining the multiple-scales technique of Amundsen et al. [3] with the boundary layer analysis of Angell and Olmstead [4], giving a slight extension of both. The method is flexible and has the potential to be applied not only to Fredholm type integral terms but Volterra ones as well.

\subsection{Open Questions and Future Work}

1. Perhaps the most immediate, tractably pursuable future work arising from this study is the steady-state bifurcation pictures in terms of the detuning parameter $\Delta$. There is a bifurcation program, AUTO 97, used in differential equations that is capable of generating bifurcation diagrams for equations with multiple solutions which was employed by Amundsen et al. [3]. While not generally applicable to integro-differential equations, in our case the Fourier formulation provides a clear avenue to treating the KdV-Fredholm equation with AUTO 97.

2. An interesting result of the derivation was that there does not appear to be any readily identifiable coupling effects between the horizontal and bottom forcing at the fundamental frequency to this order of approximation. An interesting question is how exactly do these coupling effects, assuming they exist, manifest and what scalings are needed to have them represented in the leading order balance.

3. The main mathematical difference in the analysis of the strong forced KdV-Fredholm and the fKdV was the possibility of two negative non-dispersive solutions arising from the leading order integral equation. The open question here is what are the full implications of this possibility. This is tied into the bifurcation analysis with AUTO which we would expect would provide some insight.

4. In terms of theory development of nonlinear resonant sloshing, it seems possible that our derivation technique can be used on a pitching system with the coordinates fixed to the tank. The open question is whether or not this would lead to an analytically tractable model equation and if so, how well does it predict the observed wave behaviour. 


\section{Appendix A}

\section{Numerical Method}

In this Appendix we describe the numerical scheme for solving the full $\mathrm{KdV}$-Fredholm equation (3.3) in its Fourier formulation, written for cleanliness as

$$
\partial_{t} u-\lambda \partial_{x}^{3} u+\frac{3}{2} u \partial_{x} u-\frac{2 \nu}{i \pi}\left(\hat{u}(-1, t) e^{i \pi x}-\hat{u}(1, t) e^{-i \pi x}\right)-\mu \partial_{x}^{2} u-\pi \sin \pi x=0 .
$$

This can be treated numerically by a pseudo-spectral method originally proposed by Fornberg and Whitham [15] and employed by Cox and Mortell [12] which we follow. The method involves transforming the equation into Fourier space, time-stepping in this space, and inverting the solution using the inverse Fourier transform to find the solution. To do so we choose a uniformly grid with spacing $\Delta x=\frac{2}{N}$, where $N$ is an even integer. Defining the discrete Fourier transform as

$$
\hat{u}(W, t)=\mathcal{F}\{u\}(W, t)=\sum_{j=0}^{N-1} u(j \Delta x, t) e^{-\frac{2 \pi i W_{j}}{N}}
$$

we can transform (A.1) into discrete Fourier space giving

$$
\begin{aligned}
\partial_{t} \hat{u}+i \pi^{3} W^{3} \lambda \hat{u}-i \pi W \frac{3}{4} \mathcal{F}\left\{u^{2}(x, t)\right\}-\frac{\nu}{\pi i}\left(\delta_{1} \hat{u}(-1, t)-\delta_{-1} \hat{u}(-1, t)\right) \\
+\pi^{2} W^{2} \mu \hat{u}-\frac{\pi}{2}\left(\delta_{1}-\delta_{-1}\right)=0 .
\end{aligned}
$$

This can now be stepped in time using standard techniques for numerical solution of ODE's; in our case we choose the classic Runge-Kutta 4 [33] and inverted to recover the solution in the time-domain using the discrete inverse Fourier transform

$$
u(j \Delta x, t)=\mathcal{F}^{-1}\{\hat{u}\}=\frac{1}{N} \sum_{W=-N / 2}^{N / 2} \hat{u}(W, t) e^{\frac{2 \pi i W_{j}}{N}}
$$




\section{Bibliography}

[1] M. Abramowitz and I. A. Stegun. Handbook of mathematical functions with formulas, graphs, and mathematical tables. Dover, 1965.

[2] D. J. Acheson. Elementary fluid dynamics. Oxford Applied Mathematics and Computing Science Series. The Clarendon Press Oxford University Press, New York, 1990.

[3] D. E. Amundsen, E. A. Cox, and M. P. Mortell. Asymptotic analysis of steady solutions of the KdVB equation with application to resonant sloshing. Zeitschrift für Angewandte Mathematik und Physik, 58(6):1008-1034, 2007.

[4] J. Angell and W. Olmstead. Singularly Perturbed Volterra Integral-Equations. SIAM J. Appl. Math., 47(1):1-14, 1987.

[5] J. Angell and W. Olmstead. Singularly Perturbed Volterra Integral-Equations II. SIAM J. Appl. Math., 47(6):1150-1162, 1987.

[6] G. B. Arfken and H. J. Weber. Mathematical Methods for Physicists. Harcourt/Academic Press, Burlington, MA, 2001.

[7] C. M. Bender and S. A. Orszag. Advanced mathematical methods for scientists and engineers. I. Springer-Verlag, New York, 1999.

[8] N. R. Canada. Energy sector.

[9] W. Chester. Resonant Oscillations of Water Waves I Theory. Proc. R. Soc. Lond. Ser. A Math. Phys. Eng. Sci., 306(1484):5-\&, 1968.

[10] W. Chester and J. Bones. Resonant Oscillations of Water Waves II Experiment. Proc. R. Soc. Lond. Ser. A Math. Phys. Eng. Sci., 306(1484):23-\&, 1968. 
[11] M. W. Coffey. On series expansions giving closed-form solutions of Korteweg-de Vries-like equations. SIAM J. Appl. Math., 50(6):1580-1592, 1990.

[12] E. A. Cox and M. P. Mortell. The evolution of resonant water-wave oscillations. Journal of Fluid Mechanics, 162:99-116, 1986.

[13] O. Faltinsen and A. Timokha. Asymptotic modal approximation of nonlinear resonant sloshing in a rectangular tank with small fluid depth. Journal of Fluid Mechanics, 470:319-357, 2002.

[14] O. Faltinsen and A. Timokha. Sloshing. Cambridge University Press, New York, 2009.

[15] B. Fornberg and G. Whitham. Numerical and Theoretical Study of Certain Nonlinear Wave Phenomena. Philosophical Transactions of the Royal Society of London A, 289(1361):373-404, 1978.

[16] F. Fucito, A. Gamba, M. Martinelli, and O. Ragnisco. Nonlinear WKB Analysis of the String Equation. International Journal of Modern Physics B, 6(11-12):2123$2147,1992$.

[17] D. D. Ganji, G. A. Afrouzi, H. Hosseinzadeh, and R. A. Talarposhti. Application of homotopy-perturbation method to the second kind of nonlinear integral equations. Physics Letters A, 371(1-2):20-25, 2007.

[18] I. S. Gradstein and I. M. Ryshik. Table of Integrals, Series and Products. Academic Press, 1994.

[19] S. Grilli, P. Guyenne, and F. Dias. A fully non-linear model for three-dimensional overturning waves over an arbitrary bottom. International Journal for Numerical Methods in Fluids, 35(7):829-867, 2001.

[20] W. Hereman, P. P. Banerjee, A. Korpel, G. Assanto, A. Van Immerzeele, and A. Meerpoel. Exact solitary wave solutions of nonlinear evolution and wave equations using a direct algebraic method. J. Phys. A, 19(5):607-628, 1986.

[21] M. A. Johnson and K. Zumbrun. Rigorous Justification of the Whitham Modulation Equations for the Generalized Korteweg-de Vries Equation. Studies in Applied Mathematics, 125(1):69-89, 2010. 
[22] J. Kevorkian and J. D. Cole. Multiple Scale and Singular Perturbation Methods. Applied Mathematical Sciences. Springer-Verlag, New York, 1996.

[23] J. J. Kobine. Nonlinear resonant characteristics of shallow fluid layers. Philosophical Transactions of the Royal Society A, 366(1868):1331-1346, 2008.

[24] G. Kuzmak. Asymptotic solutions of nonlinear second order differential equations with variable coefficients. Journal of Applied Mathematics and Mechanics, 23:730$744,1959$.

[25] J. C. Luke. A perturbation method for nonlinear dispersive wave problems. Proc. Roy. Soc. Ser. A, 292:403-412, 1966.

[26] C. Mei. Applied Dynamics of Ocean Surface Waves (Advanced Series on Ocean Engineering, V. 1). World Scientific Pub Co Inc, 2 edition.

[27] R. M. Miura and M. D. Kruskal. Application of a non linear wkb method to the korteweg-devries equation. SIAM Journal on Applied Mathematics, 26(2):pp. 376395, 1974.

[28] N. Moiseyev. On the theory of nonlinear vibrations of a liquid of finite volume. Prikl. Mat. Mech., 22(612-621), 1958.

[29] S. Myint and R. Grimshaw. The Modulation of Nonlinear Periodic Wave-trains by Dissipative Terms in the Korteweg-deVries Equation. Wave Motion, 22(2):215-238, 1995.

[30] H. Ockendon and J. Ockendon. Resonant Surface Waves. Journal of Fluid Mechanics, 59:397-413, 1973.

[31] H. Ockendon, J. Ockendon, and A. Johnson. Resonant Sloshing in Shallow Water. Journal of Fluid Mechanics, 167:465-479, 1986.

[32] H. Ockendon, J. Ockendon, and D. Waterhouse. Multi-mode resonances in fluids. Journal of Fluid Mechanics, 315:317-344, 1996.

[33] A. Quarteroni, R. Sacco, and F. Saleri. Numerical Mathematics. Springer, 2007.

[34] U. Secretariat. Review of maritime transport. Technical report, United Nations Conference on Trade and Development, 2006. 
[35] G. P. Tolstov. Fourier series. Dover Publications Inc., New York, 1976.

[36] P. Trinh. Unifying a the resonant solutions of a broad class of korteweg-devries equations. Master's thesis, Carleton University, 2007.

[37] G. B. Whitham. A general approach to linear and non-linear dispersive waves using a Lagrangian. J. Fluid Mech., 22:273-283, 1965.

[38] G. B. Whitham. Linear and Nonlinear Waves. Pure and Applied Mathematics. John Wiley \& Sons Inc., New York, 1974. 